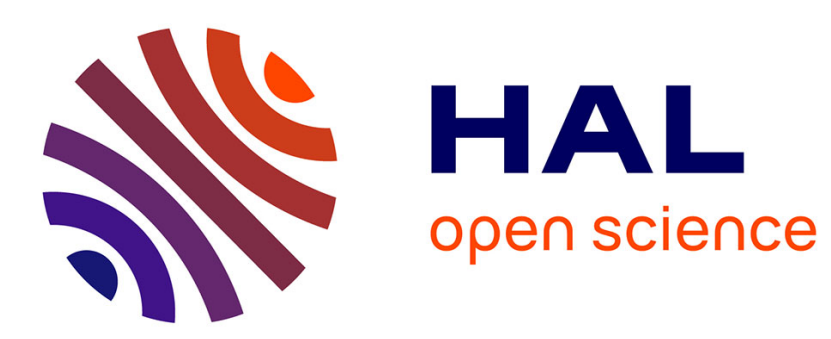

\title{
Blossoming and Hermite-Padé Approximation for Hypergeometric Series
}

\author{
Rachid Ait-Haddou, Marie-Laurence Mazure
}

\section{To cite this version:}

Rachid Ait-Haddou, Marie-Laurence Mazure. Blossoming and Hermite-Padé Approximation for Hypergeometric Series. Numerical Algorithms, 2021, 88, pp.1183-1214. 10.1007/s11075-021-01071-3 . hal-02996917

\section{HAL Id: hal-02996917 \\ https://hal.science/hal-02996917}

Submitted on 9 Nov 2020

HAL is a multi-disciplinary open access archive for the deposit and dissemination of scientific research documents, whether they are published or not. The documents may come from teaching and research institutions in France or abroad, or from public or private research centers.
L'archive ouverte pluridisciplinaire HAL, est destinée au dépôt et à la diffusion de documents scientifiques de niveau recherche, publiés ou non, émanant des établissements d'enseignement et de recherche français ou étrangers, des laboratoires publics ou privés. 


\title{
Blossoming and Hermite-Padé Approximation
}

\section{for Hypergeometric Series}

\author{
Rachid Ait-Haddou ${ }^{1}$ and Marie-Laurence Mazure ${ }^{2}$ \\ ${ }^{1}$ Department of Mathematics and Statistics, King Fahd University \\ of Petroleum \& Minerals, Dhahran 31261, Saudi Arabia \\ ${ }^{2}$ Marie-Laurence Mazure, Laboratoire Jean Kuntzmann, CNRS, Université \\ Grenoble Alpes, 38000 Grenoble, France \\ rachid.aithaddou@kfupm.edu.sa,marie-laurence.mazure@univ-grenoble-alpes.fr
}

\begin{abstract}
Based on the blossoming theory, in this work we develop a new method for deriving Hermite-Padé approximants of certain hypergeometric series. Its general principle consists in building identities generalising the Hermite identity for exponentials, and in then applying their blossomed versions to appropriate tuples to simultaneously produce explicit expressions of the approximants and explicit integral representations of the corresponding remainders. For binomial series we use the classical blossoms while for $q$-hypergeometric series we have to use $q$-blossoms.
\end{abstract}

Keywords Rational approximation · Hermite-Padé approximation · hypergeometric series $\cdot$ Hermite identity $\cdot$ blossoms $\cdot q$-blossoms

Mathematics Subject Classification (2000) 65D17 - 65D20 - 41A20 - 41A21 .

$41 \mathrm{~A} 28$

\section{Introduction}

In his seminal work on rational approximation of the exponential function [16], Hermite proved the following identity, usually referred to as the Hermite identity:

Any univariate complex polynomial $P$ satisfies

$$
G_{P}(x) e^{x}-G_{P}(0)=x \int_{0}^{1} P(t x) e^{t x} d t, \quad x \in \mathbb{C},
$$

where the polynomial $G_{P}$ is defined by

$$
G_{P}(x)=\sum_{k=0}^{+\infty}(-1)^{k} P^{(k)}(x) .
$$

From (1.1), Hermite derived simultaneous rational approximants (type II HermitePadé approximants) to a sequence of exponential functions, which enabled him to achieve the first proof of the transcendence of the number $e$. Hermite-Padé approximation of the exponential has many important applications in applied analysis, in 
particular concerning the stability of numerical methods and the efficient computation of matrix exponentials. clearly

As a special case, take $P(x)=\frac{(u-x)^{N}}{N !}$ for some $u \in \mathbb{C}$ and some integer $N$. Then,

$$
G_{P}(x)=F_{N}(u-x), \quad x \in \mathbb{C},
$$

where $F_{N}$ is the degree $N$ truncation of the exponential function, i.e.,

$$
F_{N}(x)=\sum_{k=0}^{N} \frac{x^{k}}{k !}, \quad x \in \mathbb{C} .
$$

Out of linearity, the Hermite identity (1.1) can equivalently be stated as follows:

For any non-negative integer $N$ and any $u, x \in \mathbb{C}$,

$$
F_{N}(u-x) e^{x}-F_{N}(u)=\frac{(-1)^{N} x}{N !} \int_{0}^{1}(x t-u)^{N} e^{x t} d t .
$$

Of course it even suffices to write (1.4) for any one given $u$. Now, apart from the trivial case $x=0$, for a given $N,(1.4)$ can be considered as the equality between two polynomials of exact degree $N$ in the variable $u$, depending on the parameter $x$. It is well known that this equality is equivalent to the equality of their blossoms in $N$ variables. Accordingly, we can eventually replace (1.1) by the equivalent statement:

For any non-negative integer $N$ and any $u_{1}, \ldots, u_{n}, x \in \mathbb{C}$,

$$
f_{N}\left(u_{1}-x, \ldots, u_{N}-x\right) e^{x}-f_{N}\left(u_{1}, \ldots, u_{N}\right)=\frac{(-1)^{N} x}{N !} \int_{0}^{1} e^{x t} \prod_{i=1}^{N}\left(x t-u_{i}\right) d t .
$$

where $f_{N}$ is the blossom in $N$ variables of the degree $N$ truncation (1.3). For the precise meaning of blossoms, readers are referred to Subsection 2.1. At this stage, we only need to indicate that blossoms are crucial tools of computer aided geometric design which permit efficient and very elegant handling of parametric polynomial (and polynomial spline) curves [27,20,21].

The two identities (1.4) and (1.5) can thus be considered as alternatives to the initial Hermite identity (1.1). Our attention is more specifically turned to the blossomed one (1.5), that we now consider as the "true" Hermite identity for the exponential function. It was introduced in [1], where the first author showed that blossoms can also be elegant and efficient tools for calculating some Hermite-Padé approximants. Indeed, it suffices to apply the Hermite identity (1.5) to appropriate $N$-tuples to obtain all Padé approximants to the exponential, as well as all type II Hermite-Padé approximants to a family of exponentials. These approximants are therefore expressed in terms of blossomed truncations of the Maclaurin expansion of the exponential.

In quantum calculus, the analogues of the exponential are the $q$-exponentials, simply obtained by replacing ordinary factorials by $q$-factorials, where $q$ is any real number. In [1], the Hermite identity (1.5) was extended to the $q$-exponential function, involving $q$-blossoms instead of ordinary ones (see Subsection 4.2 and [29]). They were then used to provide explicit expressions in terms of $q$-blossoms for all 
its Padé approximants [6] and all type II Hermite-Padé approximants to a family of $q$-exponentials.

As long as someone deals with polynomials, they are implicitly dealing with (polynomial) blossoms. Our main motivation in the present work is to confirm the remarkable ability of blossoms to build interesting bridges between geometric design and apparently totally separate mathematical domains: here, Padé approximation. This is why, in keeping with the methodology initiated in [1], in Section 3 we establish Hermite identities similar to (1.4) for binomial functions, and their blossomed versions. Applied to appropriate $N$-tuples, these identities will produce $[m / n]$ Padé approximants, as well as two kinds of type II Hermite-Padé approximants to sequences of binomial functions $\left(f_{1}, \ldots, f_{m}\right)$ : firstly, any $\left(n_{0}, n, \ldots, n\right)$-approximants when the functions are defined by means of pairwise distinct non-zero numbers $\lambda_{1}, \ldots, \lambda_{m}$ as

$$
f_{i}(x):=\left(1-\lambda_{i} x\right)^{\alpha}, \quad i=1, \ldots, m ;
$$

secondly, any $\left(n_{0}, n_{1}, \ldots, n_{m}\right)$-approximants when the functions depend on the previous sequence of integers as follows:

$$
f_{i}(x):=\left(1-\lambda_{i} x\right)^{\alpha-n_{i}}, \quad i=1, \ldots, m,
$$

In all cases, the approximants are therefore obtained in terms of blossomed truncations of binomial Maclaurin expansions.

Following again the same methodology as in [1], in Section 5 we extend the previous results to the quantum counterparts of binomial functions, namely all $q$ hypergeometric functions ${ }_{1} \phi_{0}(\alpha ;-; q,$.$) . The key-point consists again in finding Her-$ mite identities associated with any such $q$-hypergeometric functions, in this case stated in terms of $q$-blossoms. As previously, applied to appropriate tuples, they produce $[m / n]$-Padé approximants, and type II Hermite-Padé approximants similar to the binomial case, namely, either $\left(n_{0}, n, \ldots, n\right)$-approximants with

$$
f_{i}(x):={ }_{1} \phi_{0}\left(\alpha ;-; q, \lambda_{i} x\right), \quad i=1, \ldots, m ;
$$

or $\left(n_{0}, n_{1}, \ldots, n_{m}\right)$-approximants with

$$
f_{i}(x):={ }_{1} \phi_{0}\left(q^{n_{i}} \alpha ;-; q, \lambda_{i} x\right), \quad i=1, \ldots, m .
$$

Padé approximation for binomial functions has a long history with fundamental applications in number theory. The first to obtain explicit expressions was Thue in $[31,32]$. Later, in [28] Seigel identified these approximants (denominators and numerators) as hypergeometric polynomials of the form ${ }_{2} F_{1}$. They were then used by Baker to provide the first spectacular effective irrationality measures for some algebraic numbers such as $\sqrt[3]{2}$ [4]. Quite a number of articles have addressed the question of type II Hermite-Padé approximation for various classes of hypergeometric and $q$ hypergeometric functions, e.g., $[10,30,8,9,15]$. A common strategy to obtain type II Hermite-Padé approximants for either hypergeometric or $q$-hypergeometric series defined in a more general way than usual, was presented in [19], see also [23]. It applies in particular to (1.6) and (1.8), with however a slight limitation on the integers $n_{0}, n$. 
Though not developed here in its full generality, our blossoming approach gives further insight in the subject. Its forcefulness lies in the fact that it naturally enables us to achieve not only new explicit interpretations of existing Hermite-Padé approximants, new approximants (situations (1.7) and (1.9) depicted above), but also, simultaneously, explicit integral representations of the corresponding remainders, which is especially crucial for arithmetic applications [4,11,23]. Moreover, the fact that all the approximants are obtained as specific values of some blossoms explains Seigel's result in [28] as a straightforward consequence of the definition of blossoms. For similar reasons, we can extend it to the quantum context: the Padé approximants to any $q$-hypergeometric functions ${ }_{1} \phi_{0}$ (denominators and numerators) are $q$-hypergeometric polynomials of the form ${ }_{2} \phi_{1}$.

\section{Preliminaries}

As indicated in its title, this article establishes a link between blossoms and Padé approximants. We therefore have to start by introducing these tools, and this is the object of the present section.

\subsection{Blossoms: brief overview and preliminary results}

In this section we present a condensed overview on the definition of blossoms and classical associated tools $[27,20,21]$. We also state a number of preliminary properties which can be straightforwardly derived from their definitions. This is why the elementary proofs are left to the readers. Our purpose in gathering them separately is to avoid distracting readers from the guiding line of the proofs in the next section.

Blossoming is a fundamental concept in computer-aided geometric design, first introduced in [27] by L. Ramshaw within the polynomial framework, and then extended to the more general Chebyshevian framework in [26,22,3]. From now on, to emphasise the geometric design nature of blossoms, variables and parameters will be real numbers, though the results can be adapted to complex numbers. From now on too, $x^{[k]}$ will stand for $x$ repeated $k$ times, and, for $a, b \in \mathbb{R}$, the notation $(a, b)$ will always be used with the meaning of the element $(a, b) \in \mathbb{R}^{2}$.

Definition 2.1 Let $P$ be a real polynomial of degree at most $N$. There exists a unique muti-affine symmetric function $p$ defined on $\mathbb{R}^{N}$ such that $p\left(x^{[N]}\right)=P(x)$ for any real number $x$. The function $p$ is termed the blossom (in $N$ variables) of the polynomial $P$.

When the polynomial $P$ is expressed in the monomial basis as $P(x)=\sum_{k=0}^{N} a_{k} x^{k}$, its blossom is explicitly given by

$$
p\left(x_{1}, \ldots, x_{N}\right)=\sum_{k=0}^{N} \frac{a_{k}}{\left(\begin{array}{c}
N \\
k
\end{array}\right)} \sigma_{k}\left(x_{1}, \ldots, x_{N}\right),
$$

where $\sigma_{k}$ refers to the $k$ th elementary symmetric function

$$
\sigma_{k}\left(x_{1}, \ldots, x_{k}\right)=\sum_{1 \leq i_{1}<i_{2}<\ldots<i_{k} \leq N} x_{i_{1}} x_{i_{2}} \ldots x_{i_{k}} .
$$


Any two points $\left(a^{[N]}\right),\left(b^{[N]}\right), a \neq b$, on the diagonal of $\mathbb{R}^{N}$, can be joined by a sequence of points in $\mathbb{R}^{N}$ obtained by repeatedly replacing one copy of $a$ by $b$, thus providing the intermediate $N$-tuples $\left(a^{[N-i]}, b^{[i]}\right), i=1, \ldots, N-1$. For each $P \in \mathbb{P}_{N}$, with blossom $p$, the real numbers

$$
p_{i}:=p\left(a^{[N-i]}, b^{[i]}\right), \quad i=0, \ldots, N,
$$

are called the Bézier coefficients of $P$. The multi-affinity and the symmetry of $p$ enable us to compute each value $p\left(u_{1}, \ldots, u_{N}\right)$ of the blossom $p$ as an affine combination of the Bézier coefficients $p_{0}, \ldots, p_{N}$, with coefficients independent of $P$, through an $N$-step triangular algorithm (de Casteljau algorithm). The classical design algorithms are all contained in this fact, and this explains the importance of blossoms in CAGD. In particular on the diagonal, we obtain

$$
P(x)=p\left(x^{[N]}\right)=\sum_{k=0}^{N} p_{k} B_{k}^{N}(x,(a, b)), \quad \sum_{k=0}^{N} B_{k}^{N}(x,(a, b))=1, \quad x \in \mathbb{R},
$$

where the coefficients $B_{k}^{N}(x,(a, b)), k=0, \ldots, N$, are independent of $P$. This shows that the $(N+1)$ functions $B_{k}^{N}(.,(a, b)), k=0, \ldots, N$, form a basis of the degree $N$ polynomial space, called the Bernstein basis relative to $(a, b)$. Examining the coefficients involved in the triangular scheme leading to (2.2), it is easy to obtain the well-known expressions

$$
B_{k}^{N}(x,(a, b))=\left(\begin{array}{l}
N \\
k
\end{array}\right)\left(\frac{x-a}{b-a}\right)^{k}\left(\frac{b-x}{b-a}\right)^{N-k}, \quad k=0,1, \ldots, N .
$$

Remark 2.1 It is worthwhile mentioning that, in case $P$ is expanded as $P(x)=\sum_{k=0}^{N} a_{k} \frac{x^{k}}{k !}$, then the expression (2.1) becomes

$$
\begin{aligned}
p\left(x_{1}, \ldots, x_{N}\right) & =\sum_{k=0}^{N} \frac{a_{k}}{N(N-1) \ldots(N-k+1)} \sigma_{k}\left(x_{1}, \ldots, x_{N}\right), \\
& =\sum_{k=0}^{N} \frac{(-1)^{k} a_{k}}{(-N)_{k}} \sigma_{k}\left(x_{1}, \ldots, x_{N}\right)
\end{aligned}
$$

where, for each non-negative integer $n$, the notation $(a)_{n}$ stands for the Pochhammer symbol

$$
(a)_{n}:=a(a+1)(a+2) \ldots(a+n-1), \quad \text { with in particular }(a)_{0}:=1 .
$$

Example 2.1 It is not always necessary to derive blossoms from the expansions in the monomial basis. Indeed, an obvious useful observation is that any symmetric multi-affine function $f$ in $N$ variables is the blossom of the polynomial $F$ obtained by restriction of $f$ to the diagonal of $\mathbb{R}^{N}$. As a trivial example, the symmetric multi-affine function $f\left(x_{1}, \ldots, x_{N}\right):=\prod_{k=1}^{N}\left(x_{k}+a\right)$ is the blossom of the polynomial $F(x):=$ $(x+a)^{N}$ since $f\left(x^{[N]}\right)=F(x)$ for all $x$. 
Let us now consider the one-to-one linear map $\Theta: \mathbb{P}_{N} \longrightarrow \mathbb{P}_{N}$ defined by

$$
\Theta(P)(x):=(1-x)^{N} P\left(\frac{x}{1-x}\right), \quad x \in \mathbb{R}, x \neq 1,
$$

for each $P \in \mathbb{P}_{N}$. If $Q=\Theta(P)$, we similarly have

$$
P(u)=(1+u)^{N} Q\left(\frac{u}{1+u}\right), \quad u \in \mathbb{R}, u \neq-1 .
$$

Proceeding as indicated in Example 2.1 we can state

Proposition 2.1 Given $P \in \mathbb{P}_{N}$, consider the bivariate polynomial $H(.,$.$) such that,$ for all $x, u \in \mathbb{R}$, with $x \neq 1$,

$$
H(x, u):=(1-x)^{N} P\left(\frac{x+u}{1-x}\right) .
$$

Then, for each $x, u \in \mathbb{R}$, the blossoms $h(x ;$.$) and h(. ; u)$ (in $N$ variables) of the polynomials $H(x ;$.$) and H(. ; u)$, respectively, are the continuous functions defined in terms of the blossom $p$ of $P$ by the expressions

$$
\begin{aligned}
& h\left(x ; u_{1}, \ldots, u_{N}\right)=(1-x)^{N} p\left(\frac{x+u_{1}}{1-x}, \ldots, \frac{x+u_{N}}{1-x}\right), \\
& h\left(x_{1}, \ldots, x_{N} ; u\right)=\prod_{j=1}^{N}\left(1-x_{j}\right) p\left(\frac{x_{1}+u}{1-x_{1}}, \ldots, \frac{x_{N}+u}{1-x_{N}}\right),
\end{aligned}
$$

wherever they are meaningful.

Note that the bivariate polynomial $H$ defined in (2.5) interpolates between the polynomials $P$ and $Q:=\Theta(P)$ in the sense that

$$
H(0, u)=P(u) \quad \text { and } \quad H(x, 0)=Q(x) \quad \text { for all } x, u \in \mathbb{R} .
$$

Moreover, we can symmetrically derive $H$ from $Q$ as follows:

$$
H(x, u):=(1+u)^{N} Q\left(\frac{x+u}{1+u}\right), \quad x, u \in \mathbb{R}, u \neq-1 .
$$

Finally, the second formula in (2.6) shows that the blossom $q$ of the polynomial $Q=$ $\Theta(P)$ can be calculated from the blossom $p$ of $P$ and conversely, from

$$
\begin{aligned}
& q\left(x_{1}, \ldots, x_{N}\right)=p\left(\frac{x_{1}}{1-x_{1}}, \ldots, \frac{x_{N}}{1-x_{N}}\right) \prod_{j=1}^{N}\left(1-x_{j}\right), \\
& p\left(u_{1}, \ldots, u_{N}\right)=q\left(\frac{u_{1}}{1+u_{1}}, \ldots, \frac{u_{N}}{1+u_{N}}\right) \prod_{j=1}^{N}\left(1+u_{j}\right) .
\end{aligned}
$$

We conclude this section with a useful reminder on how to decrease the degree of polynomials in the blossoming approach. The most natural way to linearly map $\mathbb{P}_{N}$ onto $\mathbb{P}_{N-1}$ is the subblossoming principle obtained by fixing one variable inside the 
blossoms. More precisely, with each $P \in \mathbb{P}_{N}$, one can associate the function $\widetilde{P} \in \mathbb{P}_{N-1}$ defined by $\widetilde{P}(x):=p\left(a, x^{[N-1]}\right)$, where $p$ is the blossom of $P$, and $a$ is a given real number. The blossom $\widetilde{p}$ of $\widetilde{P}$ is then defined by $\widetilde{p}\left(x_{1}, \ldots, x_{N-1}\right):=p\left(a, x_{1}, \ldots, x_{N-1}\right)$. The process can of course be repeated: in particular, when inserting $i$ times 0 inside the blossoms, the following result readily follows from (2.1).

Proposition 2.2 Let $p$ be the blossom in $N$ variables of a given polynomial $P \in \mathbb{P}_{N}$, expanded in the monomial basis as $P_{N}(x)=\sum_{k=0}^{N} a_{k} x^{k}$. Then, the polynomial function $\widetilde{P}$ of degree at most $(N-i)$ defined by

$$
\widetilde{P}(x):=p\left(0^{[i]}, x^{[N-i]}\right), \quad x \in \mathbb{R} .
$$

is expanded as

$$
\widetilde{P}(x)=\sum_{k=0}^{N-i} \widetilde{a}_{k} x^{k}, \quad \text { with } \widetilde{a}_{k}=a_{k} \frac{(-N+i)_{k}}{(-N)_{k}} \text { for } k=0, \ldots, N-i .
$$

\subsection{Padé approximants}

The definition of Hermite-Padé approximants slightly differs from one author to another $[5,7,33,34]$. We therefore have to specify the one we will use in this work, especially well-suited for functions which do not vanish at the origin.

Definition 2.2 Let $f$ be a real function in one variable which is analytic close to the origin and which satisfies $f(0)=1$. Given two non-negative integers $m, n$, and any real polynomials $P_{m}, Q_{n}$, we say that the couple $\left(P_{m}, Q_{n}\right)$ is an $[m / n]$-Padé approximant to $f$ if the following properties are satisfied:

a) $Q_{n}$ if of degree at most $n$ and $Q_{n}(0)=1$;

b) $P_{m}$ if of degree at most $m$;

c) the remainder $R_{m, n}:=Q_{n} f-P_{m}$ vanishes at least $(N+1)$ times at the origin, where $N:=m+n$.

The definition and the assumption $f(0)=1$ make it obvious that $\left(P_{m}, Q_{n}\right)$ is an $[m / n]$ Padé approximant to $f$ if and only $\left(Q_{n}, P_{m}\right)$ is an $[n / m]$-Padé approximant to the inverse of $f$. On the other hand it is well-known that the existence of such an $[m / n]$ Padé approximant is not guaranteed. The existence means that we can determine a polynomial $Q_{n}(x)=1+\sum_{i=1}^{n} X_{i} x^{i}$ so that

$$
\left(Q_{n} f\right)^{(j)}(0)=0 \quad \text { for } m+1 \leq j \leq m+n,
$$

and when this is possible, the rational function $P_{m} / Q_{n}$ is unique. Recall that, using Leibniz formulæ, the relations (2.10) can be expressed as the following linear system of $n$ equations in the $n$ unknowns $X_{1}, \ldots, X_{n}$ :

$$
\sum_{i=1}^{n} C_{j-i} X_{i}=-C_{j}, \quad j=m+1, \ldots, m+n,
$$


where, near $0, f$ is expanded as $f(x)=\sum_{i=0}^{+\infty} C_{i} x^{i}$, with $C_{0}=1$, and with the convention that $C_{i}=0$ for $i<0$. Both existence and uniqueness of the $[m / n]$-Padé approximant to $f$ are thus ensured if and on if

$$
\Delta_{m, n}(f):=\left|\begin{array}{llll}
C_{m} & C_{m-1} & \ldots & C_{m-n+1} \\
C_{m+1} & C_{m} & \ldots & C_{m-n+2} \\
& & \ddots & \\
C_{m+n-1} & C_{m+n-2} & \ldots & C_{m}
\end{array}\right| \neq 0 .
$$

Definition 2.2 extends in a natural way to simultaneous rational approximation of a family of functions as follows:

Definition 2.3 Given $m \geq 1$, let $f_{1}, \ldots, f_{m}$ be real analytic functions of one variable near the origin with , satisfying $f_{k}(0)=1$ for $k=1, \ldots, m$. Given non-negative integers $n_{0}, n_{1}, \ldots, n_{m}$, set $N=n_{0}+n_{1}+\ldots+n_{m}$. We say that a sequence $\left(P_{0}, P_{1}, \ldots, P_{m}\right)$ of real polynomials is a type II Hermite-Padé approximant to the sequence $\left(f_{1}, \ldots\right.$, $\left.f_{m}\right)$, attached to the integer sequence $\left(n_{0}, n_{1}, \ldots, n_{m}\right)$ (for short, an $\left(n_{0}, n_{1}, \ldots, n_{m}\right)$ type II Hermite-Padé approximant) when the following properties are satisfied:

a) $P_{0}(0)=1$

b) for $k=0, \ldots, m, P_{k}$ is of degree at most $\left(N-n_{k}\right)$;

c) for $k=1, \ldots, m$, the remainder $R_{k}:=P_{0} f_{k}-P_{k}$ vanishes at least $(N+1)$ times at the origin.

Here again, due to our requirement that $P_{0}(0)=1$, the existence is not guaranteed, and the search for such a simultaneous approximant consists in determining the $n=$ $\sum_{i=1}^{m} n_{i}$ unknowns $X_{1}, \ldots, X_{n}$ such that $P_{0}(x)=1+\sum_{i=1}^{n} X_{i} x^{i}$ so that the $n$ conditions

$$
\left(P_{0} f_{k}\right)^{(j)}(0)=0 \quad \text { for } N-n_{k}+1 \leq j \leq N \text { and for } k=1, \ldots, m,
$$

are satisfied. As previously, existence and uniqueness are obtained when a certain determinant similar to (2.11) is not zero. This, in general, is far from being an easy problem to solve.

Nevertheless, we are not that much interested in uniqueness, but rather in exhibiting one type II Hermite-Padé system through blossoms. In particular we have seen how to reduce the degree from $N$ to $\left(N-n_{k}\right)$ by inserting $n_{k}$ copies of 0 inside blossoms in $N$ variables. When the system $f_{1}, \ldots, f_{m}$, are variations on a fixed function $f$ (e.g., when $f_{k}(x)=f\left(\lambda_{k} x\right)$ for $k=1, \ldots, m$, the $\lambda_{k}$ being non-zero pairwise distinct numbers) it is thus natural to apply this idea to some blossomed Hermite-identity generalising (1.5) related to this function, as was done in [1] for the exponential function. This is what will be done in next section for binomial functions.

\section{Hermite identity and Padé approximants to binomial functions}

Inspired by the exponential, in this section we construct Hermite identities for binomial functions of the variable $x$. With this in view, we introduce an extra variable $u$ with respect to which these identities will be polynomial, the construction involving the correspondence $\Theta$ mentioned in the previous section. The blossomed versions of these identities will then naturally generate Hermite-Padé approximants. 
3.1 Hermite identities for binomial functions

As is well know, the Pochhammer symbols (2.4) permit condensed expressions for the Maclaurin series of a number of classical functions. The basic example is the binomial function

$$
(1-x)^{-\alpha}=\sum_{k=0}^{+\infty}(\alpha)_{k} \frac{x^{k}}{k !}, \quad|x|<1,
$$

where $\alpha$ is a given real number. Subsequently, let $N$ be a given non-negative integer. Then, denoting by $Q_{N}^{\alpha}$ the degree $N$ truncation of this series, the previous identity yields

$$
(1-x)^{-\alpha}=Q_{N}^{\alpha}(x)+O\left(x^{N+1}\right) \quad \text { as } x \rightarrow 0 .
$$

Replacing $x$ by $u /(1+u)$ and multiplying both sides by $(1+u)^{N}$, this yields

$$
(1+u)^{N+\alpha}=(1+u)^{N} Q_{N}^{\alpha}\left(\frac{u}{1+u}\right)+O\left(u^{N+1}\right) \quad \text { as } u \rightarrow 0 .
$$

Accordingly, the polynomial $P_{N}^{\alpha} \in \mathbb{P}_{N}$ which satisfies

$$
Q_{N}^{\alpha}=\Theta\left(P_{N}^{\alpha}\right)
$$

is the degree $N$ truncation of the Maclaurin series of $\left(1+{ }^{-}\right)^{N+\alpha}$. Now, for $|u|<1$,

$$
(1+u)^{N+\alpha}=\sum_{k=0}^{+\infty} A_{k} \frac{u^{k}}{k !}, \quad \text { with } A_{k}:=(-1)^{k}(-N-\alpha)_{k} \text { for all } k \geq 0 .
$$

The left identity in (3.1) can be written as

$$
(1+u)^{N+\alpha}=P_{N}^{\alpha}(u)+R_{N}^{\alpha}(u), \quad|u|<1,
$$

with

$$
P_{N}^{\alpha}(u)=\sum_{k=0}^{N} A_{k} \frac{u^{k}}{k !} \text { for any } u \in \mathbb{R}, \quad R_{N}^{\alpha}(u)=\sum_{k=N+1}^{+\infty} A_{k} \frac{u^{k}}{k !} \text { for }|u|<1 .
$$

Observe that

$$
Q_{N}^{\alpha}(0)=P_{N}^{\alpha}(0)=1 .
$$

With the notations introduced above, we first prove the following useful lemma.

Lemma 3.1 For any real number $\alpha$, and any $x, u \in]-1,1[$ such that $2 x+u<1$, the function $R_{N}^{\alpha}$ defined in (3.3) satisfies

$$
\frac{\partial}{\partial x}\left[(1-x)^{N+\alpha} R_{N}^{\alpha}\left(\frac{x+u}{1-x}\right)\right]=\frac{A_{N+1}}{N !}(x+u)^{N}(1-x)^{\alpha-1} .
$$


Proof The requirements on $x, u$ ensure that $\left|\frac{x+u}{1-x}\right|<1$. For the sake of simplicity, let us set

$$
\vartheta(x):=\frac{x+u}{1-x}, \quad \text { so that } \vartheta^{\prime}(x):=\frac{1+\vartheta(x)}{1-x} .
$$

With this notation, we thus have

$$
\begin{aligned}
\frac{\partial}{\partial x}\left[(1-x)^{N+\alpha} R_{N}^{\alpha}(\vartheta(x))\right]=(1-x)^{N+\alpha-1}[- & (N+\alpha) R_{N}^{\alpha}(\vartheta(x)) \\
& \left.+(1+\vartheta(x)) R_{N}^{\alpha \prime}(\vartheta(x))\right] .
\end{aligned}
$$

Now, for any $|y|<1$,

$$
\begin{aligned}
-(N+\alpha) R_{N}^{\alpha}(y)+(1+y) R_{N}^{\alpha \prime}(y) & =A_{N+1} \frac{y^{N}}{N !}+\sum_{k=N+1}^{+\infty}\left[A_{k+1}+(k-N-\alpha) A_{k}\right] \frac{y^{k}}{k !}, \\
& =A_{N+1} \frac{y^{N}}{N !}
\end{aligned}
$$

on account of (3.1). The claim (3.4) readily follows.

The previous lemma will serve in Theorem 3.1 below to provide a Hermite identity associated with any given binomial function $(1-x)^{\alpha}$. This identity involves the bivariate polynomial $H_{N}^{\alpha}$ associated with $P_{N}^{\alpha}$ as in the previous subsection, from the expression

$$
H_{N}^{\alpha}(x, u)=(1-x)^{N} P_{N}^{\alpha}\left(\frac{x+u}{1-x}\right), \quad \text { for } x, u \in \mathbb{R}, x \neq 1 .
$$

Theorem 3.1 Given any real number $\alpha$, we have, for any $x \in]-1,1[$ and any $u \in \mathbb{R}$,

$$
H_{N}^{\alpha}(x, u)(1-x)^{\alpha}-P_{N}^{\alpha}(u)=E_{N}^{\alpha}(x ; u),
$$

where

$$
E_{N}^{\alpha}(x ; u)=x \frac{(-1)^{N}(-N-\alpha)_{N+1}}{N !} \int_{0}^{1}(t x+u)^{N}(1-t x)^{\alpha-1} d t
$$

Proof Subsequently, we work under the requirements $x, u \in]-1,1[$, and $2 x+u<1$, as in Lemma 3.1. We can then successively replace $u$ by $\frac{x+u}{1-x}$ in the identity (3.2) and then multiply by $(1-x)^{N+\alpha}$. This yields

$$
(1+u)^{N+\alpha}=H_{N}^{\alpha}(x, u)(1-x)^{\alpha}+(1-x)^{N+\alpha} R_{N}^{\alpha}\left(\frac{x+u}{1-x}\right) .
$$

The left-hand side of the previous identity does not depend on $x$. Therefore, taking account of (3.4) we obtain

$$
\frac{\partial}{\partial x}\left[H_{N}^{\alpha}(x, u)(1-x)^{\alpha}\right]=-\frac{A_{N+1}}{N !}(x+u)^{N}(1-x)^{\alpha-1} .
$$


Let us introduce an additional variable $t \in[0,1]$. Then, the previous relation leads to:

$$
\frac{\partial}{\partial t}\left[H_{N}^{\alpha}(t x, u)(1-t x)^{\alpha}\right]=-x \frac{A_{N+1}}{N !}(t x+u)^{N}(1-t x)^{\alpha-1}, \quad t \in[0,1] .
$$

Let us now integrate in the variable $t$ between 0 and 1 . Taking account of the interpolating property (2.7) of $H_{N}^{\alpha}$, this yields

$$
H_{N}^{\alpha}(x, u)(1-x)^{\alpha}-P_{N}^{\alpha}(u)=-x \frac{A_{N+1}}{N !} \int_{0}^{1}(t x+u)^{N}(1-t x)^{\alpha-1} d t .
$$

Given that $A_{N+1}$ is defined according to (3.1), this identity is exactly (3.6). However, a priori we have proved its validity only under the initial requirements $x, u \in]-1,1$, $2 x+u<1$. Nonetheless, for any given $x \in]-1,1$, the expressions involved in both sides of (3.6) are polynomials of degree at most $N$ in the variable $u$, therefore defined for any $u \in \mathbb{R}$. Since they coincide for $-1<u<\min (1,1-2 x)$, they coincide on the whole of $\mathbb{R}$. This concludes the proof of Theorem 3.1 .

Below we state the blossomed version of Theorem 3.1. This blossomed Hermite identity will be the one of interest for Padé approximation.

Theorem 3.2 Given any real number $\alpha$, any $x \in]-1,1\left[\right.$, and any $u_{1}, \ldots, u_{N} \in \mathbb{R}$, we have

$$
h_{N}^{\alpha}\left(x ; u_{1}, u_{2}, \ldots, u_{N}\right)(1-x)^{\alpha}-p_{N}^{\alpha}\left(u_{1}, u_{2}, \ldots, u_{N}\right)=e_{N}^{\alpha}\left(x ; u_{1}, \ldots, u_{N}\right),
$$

where

$$
e_{N}^{\alpha}\left(x ; u_{1}, \ldots, u_{N}\right):=x \frac{(-1)^{N}(-N-\alpha)_{N+1}}{N !} \int_{0}^{1} \prod_{i=1}^{N}\left(t x+u_{i}\right)(1-x t)^{\alpha-1} d t,
$$

and where the functions $h_{N}^{\alpha}(x ;$.$) and p_{N}^{\alpha}$, defined on $\mathbb{R}^{N}$, denote the blossoms of the polynomials $H_{N}^{\alpha}(x,$.$) and P_{N}^{\alpha}$, respectively.

Proof Observe that, for a given $x \in]-1,1\left[\right.$, the function $e_{N}^{\alpha}(x ;$.$) defined on \mathbb{R}^{N}$ by (3.9) is simply obtained by blossoming the polynomial $(.+t x)^{N}$ inside the integral, according to the expression mentioned in Example 2.1. It is clearly symmetric and multi-affine in $u_{1}, \ldots, u_{N}$ and it coincides with $E_{N}^{\alpha}(x, u)$ when $u_{1}=\cdots=u_{N}=u$. So it is the blossom of the polynomial $E_{N}^{\alpha}(x,$.$) . That the left-hand side of (3.8) is the$ blossomed version of the left-hand side of (3.6) is due to the interpolating property (2.7) of $H_{N}^{\alpha}$.

\subsection{Padé Approximation for Binomial Functions}

According to Proposition 2.2, the evaluation of blossoms on $N$-tuples of the from $\left(0^{[i]}, x^{[N-i]}\right)$ maps $\mathbb{P}_{N}$ onto $\mathbb{P}_{N-i}$. With a view to build Padé approximant, it is thus natural to apply the Hermite identity (3.8) with such $N$-tuples. 
Theorem 3.3 Given any positive integers $m, n$, set $N:=m+n$. Then, for any real number $\alpha$, an $[m / n]$-Padé approximant to the binomial function $(1-x)^{\alpha}$ is given by the pair $\left(P_{m}, Q_{n}\right)$ defined by

$$
P_{m}(x)=p_{N}^{\alpha-m}\left(0^{[n]},(-x)^{[m]}\right), \quad Q_{n}(x)=q_{N}^{\alpha-m}\left(0^{[m]}, x^{[n]}\right), \quad x \in \mathbb{R},
$$

where $q_{N}^{\alpha-m}$ and $p_{N}^{\alpha-m}$ are the blossoms of the polynomials $Q_{N}^{\alpha-m}$ and $P_{N}^{\alpha-m}$ respectively. Moreover, for $|x|<1$, the quantity

$$
R_{m, n}(x):=Q_{n}(x)(1-x)^{\alpha}-P_{m}(x),
$$

is given by

$$
R_{m, n}(x)=x^{N+1} \frac{(-1)^{n}(-\alpha-n)_{N+1}}{N !} \int_{0}^{1} t^{n}(1-t)^{m}(1-x t)^{\alpha-m-1} d t .
$$

Proof Let $\beta$ be a real number. Apply the Hermite identity (3.8) with $u_{1}=u_{2}=\ldots=$ $u_{n}=0$ and $u_{n+1}=u_{n+2} \ldots=u_{N}=-x$, where $\left.x \in\right]-1,1[$. This gives:

$$
h_{N}^{\beta}\left(x ; 0^{[n]},(-x)^{[m]}\right)(1-x)^{\beta}-p_{N}^{\beta}\left(0^{[n]},(-x)^{[m]}\right)=e_{N}^{\beta}\left(x ; 0^{[n]},(-x)^{[m]}\right) .
$$

From (2.6) and (2.8), we can deduce that

$$
h_{N}^{\beta}\left(x ; 0^{[n]},(-x)^{[m]}\right)=(1-x)^{m} q_{N}^{\beta}\left(0^{[m]}, x^{[n]}\right) .
$$

Thus, from (3.12), we have

$$
q_{N}^{\beta}\left(0^{[m]}, x^{[n]}\right)(1-x)^{\beta+m}-p_{N}^{\beta}\left(0^{[n]},(-x)^{[m]}\right)=e_{N}^{\beta}\left(x ; 0^{[n]},(-x)^{[m]}\right) .
$$

Take $\beta:=\alpha-m$. Then, $e_{N}^{\beta}\left(x ; 0^{[n]},(-x)^{[m]}\right)$ is the quantity $R_{m, n}(x)$ defined in (3.11). The previous identity can thus be written as follows:

$$
q_{N}^{\alpha-m}\left(0^{[m]}, x^{[n]}\right)(1-x)^{\alpha}-p_{N}^{\alpha-m}\left(0^{[n]},(-x)^{[m]}\right)=R_{m, n}(x) .
$$

Since $Q_{n}(0)=Q_{N}^{\alpha}(0)=1$, the proof is complete.

We know that the polynomials $Q_{N}^{\alpha-m}$ and $P_{N}^{\alpha-m}$ are expanded as:

$$
Q_{N}^{\alpha-m}(x)=\sum_{k=0}^{N}(\alpha-m)_{k} \frac{x^{k}}{k !}, \quad P_{N}^{\alpha-m}(x)=\sum_{k=0}^{N}(-1)^{k}(-n-\alpha)_{k} \frac{x^{k}}{k !} .
$$

Accordingly, by straightforward application of (2.9), we can state:

Corollary 3.1 Given any non-negative integers $m, n$ and any $\alpha \in \mathbb{R}$, the $[m / n]$-Padé approximant $\left(P_{m}, Q_{n}\right)$ to the binomial function $(1-x)^{\alpha}$ defined in (3.14) can be written as follows:

$$
P_{m}(x)=\sum_{k=0}^{m} \frac{(-n-\alpha)_{k}(-m)_{k}}{(-n-m)_{k}} \frac{x^{k}}{k !}, \quad Q_{n}(x)=\sum_{k=0}^{n} \frac{(\alpha-m)_{k}(-n)_{k}}{(-n-m)_{k}} \frac{x^{k}}{k !} .
$$

Remark 3.1 It is interesting to point out a geometric design interpretation of (3.14). Indeed, for each non-zero value of $x$, the $[m / n]$-Padé approximant $\left(P_{m}, Q_{n}\right)$ is the Bézier point $g\left(0^{[m]}, x^{[n]}\right)$ of the degree $N$ polynomial planar parametric curve $G(u):=$ $\left(P_{N}^{\alpha-m}(u-x), Q_{N}^{\alpha-m}(u)\right), u \in \mathbb{R}$, relative to $(0, x)$. We can say as well that the rational approximant $P_{m} / Q_{n}$ is the corresponding rational Bézier point of the rational function $P_{N}^{\alpha-m}(u-x) / Q_{N}^{\alpha-m}(u)$. 
3.3 A glance at hypergeometric series

Recall that, with any two sequences $\left(a_{1}, \ldots, a_{r}\right),\left(b_{1}, \ldots, b_{s}\right)$, one can associate a socalled hypergeometric series [12]

$$
{ }_{r} F_{s}\left(a_{1}, \ldots, a_{r} ; b_{1}, \ldots, b_{s} ; x\right):=\sum_{k=0}^{+\infty} \frac{\left(a_{1}\right)_{k} \ldots,\left(a_{r}\right)_{k}}{\left(b_{1}\right)_{k} \ldots,\left(b_{s}\right)_{k}} \frac{x^{k}}{k !} .
$$

If no $b_{i}$ is a negative integer, all coefficients involved in this expansion are welldefined. Supposing that, for some $j \in\{1, \ldots, s\}, b_{j}=-K$ where $K$ is a positive integer, the previous series is still meaningful provided that one of the $a_{k}$ 's, say $a_{r}$, is of the form $a_{r}=-L$, where $L \leq K$ is a positive integer, under the convention that $0 / 0=0$.

The expressions (3.15) make it obvious that the $[\mathrm{m} / \mathrm{n}]$-Padé approximant to the binomial function $(1-x)^{\alpha}$ obtained in Corollary 3.1 can be described by the usual expression

$$
\left[P_{m}, Q_{n}\right]=\left[{ }_{2} F_{1}(-\alpha-n,-m ;-N ; .),{ }_{2} F_{1}(\alpha-m,-n ;-N ; .)\right],
$$

with $N:=m+n$. Moreover, using the integral representation of the Gauss hypergeometric function

${ }_{2} F_{1}(a, b ; c ; x)=\frac{\Gamma(c)}{\Gamma(b) \Gamma(c-b)} \int_{0}^{1} t^{b-1}(1-t)^{c-b-1}(1-z t)^{-a} d t, \quad \operatorname{Re}(c)>\operatorname{Re}(b)>0$,

the relation (3.14) is transformed into the classical [4]

$$
\begin{aligned}
{ }_{2} F_{1}(\alpha-m,-n ;-N ; x) & (1-x)^{\alpha}-{ }_{2} F_{1}(-\alpha-n,-m ;-N ; x) \\
& =x^{N+1} \frac{(-1)^{N}(-\alpha-n)}{(N+1) !\left(\begin{array}{l}
N \\
n
\end{array}\right)}{ }_{2} F_{1}(-\alpha+m+1, n+1 ; N+2 ; x) .
\end{aligned}
$$

As a matter of fact, the expressions (3.15) - or (3.16) as well - are special cases of a more general result concerning hypergeometric series stated below as an immediate consequence of Proposition 2.2.

Corollary 3.2 Given any integers $r, s$, let $P_{N}$ be the degree $N$ truncation of the hypergeometric series ${ }_{r} F_{S}\left(a_{1}, \ldots, a_{r} ; b_{1}, \ldots, b_{s} ;.\right)$, and let $p_{N}$ be its blossom. Then, for each $i=0, \ldots, N$,

$$
p_{N}\left(0^{[i]}, x^{[N-i]}\right)={ }_{r+1} F_{S+1}\left(a_{1}, \ldots, a_{r},-N+i ; b_{1}, \ldots, b_{s},-N ; x\right), \quad x \in \mathbb{R} .
$$

Observing that, when $x \neq 0, p_{N}\left(0^{[i]}, x^{[N-i]}\right)$ is the $i$ th Bézier coefficient of $P_{N}$ relative to $(x, 0)$, the interesting following expansion readily follows.

Corollary 3.3 With the same notations as in Corollary 3.2, let $\beta$ be any non-zero real number. Then, the truncated function $P_{N}$ can be expanded in the Bernstein basis relative to $(\beta, 0)$ as follows:

$$
P_{N}=\sum_{i=0}^{N}{ }_{r+1} F_{s+1}\left(a_{1}, \ldots, a_{r},-N+i ; b_{1}, \ldots, b_{s},-N ; \beta\right) B_{i}^{N}(.,(\beta, 0))
$$


Remark 3.2 The expansion (3.17) can also be written as follows

$$
\begin{aligned}
{ }_{r+1} F_{s+1} & \left(a_{1}, \ldots, a_{r},-N ; b_{1}, \ldots, b_{s},-N ; .\right) \\
= & \sum_{i=0}^{N} r+1 F_{s+1}\left(a_{1}, \ldots, a_{r},-N+i ; b_{1}, \ldots, b_{s},-N ; \beta\right) B_{i}^{N}(.,(\beta, 0)),
\end{aligned}
$$

the left hand-side of (3.18) being another possible expression of the truncation $P_{N}$.

3.4 Type II Hermite-Padé approximation to binomial functions

Based on the generalised Hermite identity (3.8), we shall provide explicit expressions of some type II Hermite-Padé approximants to the system

$$
\left(1-\lambda_{1} x\right)^{\alpha}, \ldots,\left(1-\lambda_{m} x\right)^{\alpha},
$$

where $\lambda_{1}, \ldots, \lambda_{m}$ are given pairwise distinct non-zero real numbers, as well as of the corresponding remainders.

Theorem 3.4 Given any two non-negative integers $n_{0}, n$, an $\left(n_{0}, n, \ldots, n\right)$-HermitePadé approximant $\left(P_{0}, P_{1}, \ldots, P_{m}\right)$ to the system (3.19) is given by

$$
P_{0}(x)=p_{N}^{-\alpha-n}\left(0^{\left[n_{0}\right]},\left(-\lambda_{1} x\right)^{[n]}, \ldots,\left(-\lambda_{m} x\right)^{[n]}\right)
$$

and

$P_{j}(x)=\left(1-\lambda_{j} x\right)^{N-n} p_{N}^{-\alpha-n}\left(\left(\frac{\lambda_{j} x}{1-\lambda_{j} x}\right)^{\left[n_{0}\right]},\left(\frac{\left(\lambda_{j}-\lambda_{1}\right) x}{1-\lambda_{j} x}\right)^{[n]}, \ldots,\left(\frac{\left(\lambda_{j}-\lambda_{m}\right) x}{1-\lambda_{j} x}\right)^{[n]}\right)$, with $N=n_{0}+n m$. Moreover, for $k=1,2, \ldots, m$, and for any $x$ such that $\left|\lambda_{j} x\right|<1$, we have

$$
\begin{aligned}
P_{0}(x)\left(1-\lambda_{j} x\right)^{\alpha}-P_{j}(x)=(-x)^{N+1} & \frac{(-N+\alpha+n)_{N+1}}{N !} \lambda_{j}^{n_{0}+1}\left(1-\lambda_{j} x\right)^{\alpha} \\
& \times \int_{0}^{1}\left(1-\lambda_{j} x t\right)^{-\alpha-n-1} t^{n_{0}} \prod_{k=1}^{m}\left(t \lambda_{j}-\lambda_{k}\right)^{n} d t .
\end{aligned}
$$

Proof Given a real number $\beta$, consider the associated generalised Hermite identity (3.8) with $N=n_{0}+m n$. Given any real number $x$ such that $\left|\lambda_{k} x\right|<1$ for $k=1, \ldots, m$, throughout the proof, the $N$-tuple $\left(u_{1}, \ldots, u_{N}\right)$ will be defined by:

$$
\left(u_{1}, \ldots, u_{N}\right):=\left(0^{\left[n_{0}\right]},\left(-\lambda_{1} x\right)^{[n]}, \ldots,\left(-\lambda_{m} x\right)^{[n]}\right) .
$$

Select an integer $j \in\{0,1, \ldots, m\}$. Then, we have

$$
h_{N}^{\beta}\left(\lambda_{j} x ; u_{1}, \ldots, u_{N}\right)\left(1-\lambda_{j} x\right)^{\beta}-p_{N}^{\beta}\left(u_{1}, \ldots, u_{N}\right)=e_{N}^{\beta}\left(\lambda_{j} x ; u_{1}, \ldots, u_{N}\right),
$$

where, according to (3.9), here the quantity $e_{N}^{\beta}\left(\lambda_{j} x, u_{1}, \ldots, u_{N}\right)$ is equal to

$$
x^{N+1} \frac{(-1)^{N}(-N-\beta)_{N+1}}{N !} \lambda_{j}^{n_{0}+1} \int_{0}^{1}\left(1-\lambda_{j} x t\right)^{\beta-1} t^{n_{0}} \prod_{k=1}^{m}\left(t \lambda_{j}-\lambda_{k}\right)^{n} d t,
$$


From (2.6) we know that

$$
\begin{aligned}
h_{N}^{\beta}\left(\lambda_{j} x ; u_{1}, \ldots, u_{N}\right)= & \left(1-\lambda_{j} x\right)^{N} \\
& \times p_{N}^{\beta}\left(\left(\frac{\lambda_{j} x}{1-\lambda_{j} x}\right)^{\left[n_{0}\right]},\left(\frac{\left(\lambda_{j}-\lambda_{1}\right) x}{1-\lambda_{j} x}\right)^{[n]}, \ldots,\left(\frac{\left(\lambda_{j}-\lambda_{m}\right) x}{1-\lambda_{j} x}\right)^{[n]}\right), \\
= & \left(1-\lambda_{j} x\right)^{\beta+n} P_{j}(x), \quad\left|\lambda_{j} x\right|<1,
\end{aligned}
$$

where the quantity $P_{j}(x)$ is defined by

$$
P_{j}(x):=\left(1-\lambda_{j} x\right)^{N-n} p_{N}^{\beta}\left(\left(\frac{\lambda_{j} x}{1-\lambda_{j} x}\right)^{\left[n_{0}\right]},\left(\frac{\left(\lambda_{j}-\lambda_{1}\right) x}{1-\lambda_{j} x}\right)^{[n]}, \ldots,\left(\frac{\left(\lambda_{j}-\lambda_{m}\right) x}{1-\lambda_{j} x}\right)^{[n]}\right) .
$$

Now, the $N$-tuple at which $p_{N}^{\beta}$ is evaluated in (3.22) contains $n$ copies of 0 . Observe that the polynomial $\widetilde{P}_{N}^{\beta}(z):=p_{N}^{\beta}\left(0^{[n]}, z^{[N-n]}\right)$ is of degree at most $(N-n)$ and its blossom in $(N-n)$ variables is given by $\widetilde{p}_{N}^{\beta}\left(z_{n+1}, \ldots, z_{N}\right):=p_{N}^{\beta}\left(0^{[n]}, z_{n+1}, \ldots, z_{N}\right)$. Applying (2.1) to the polynomial $\widetilde{P}_{N}^{\beta}$, it is easily seen that the polynomial $P_{j}$ itself belongs to $\mathbb{P}_{N-n}$. See Remark 3.3 below for its exact expression. Let us write the left-hand side of (3.20) as:

$$
\left(1-\lambda_{j} x\right)^{\beta+n} P_{j}(x)-P_{0}(x), \quad \text { with } P_{0}(x):=p_{N}^{\beta}\left(u_{1}, \ldots, u_{N}\right)
$$

Since $u_{1}=\cdots=u_{n_{0}}=0, P_{0}$ is a polynomial of degree at most $\left(N-n_{0}\right)$ in $x$. and $P_{0}(0)=P_{N}^{\beta}(0)=1$. Taking $\beta:=-\alpha-n$ and multiplying both sides in (3.20) by $-\left(1-\lambda_{j} x\right)^{\alpha}$ concludes the proof.

Remark 3.3 Using (3.3), (2.3) and (2.9), the explicit expression of the polynomial $P_{0}$ is given by

$$
P_{0}(x)=\sum_{k=0}^{N-n_{0}} \frac{(-N+n+\alpha)_{k}(-1)^{k}}{(-N)_{k}} \sigma_{k}\left(\lambda_{1}^{[n]}, \ldots, \lambda_{m}^{[n]}\right) x^{k}
$$

while the polynomials $P_{j}$, for $j=1, \ldots, m$, are given by

$P_{j}(x)=\sum_{k=0}^{N-n} \frac{(-N+n+\alpha)_{k}}{(-N)_{k}} \sigma_{k}\left(\left(\lambda_{j}\right)^{\left[n_{0}\right]},\left(\lambda_{j}-\lambda_{1}\right)^{[n]}, \ldots,\left(\lambda_{j}-\lambda_{m}\right)^{[n]}\right) x^{k}\left(1-\lambda_{j} x\right)^{N-n-k}$.

Pairwise distinct non-zero $\lambda_{1}, \ldots, \lambda_{m}$ being given again, we obtain the following result with hardly any change in the arguments.

Theorem 3.5 Given any pairwise distinct non-negative integers $n_{0}, n_{1}, \ldots, n_{m}$, and any real number $\alpha$, an $\left(n_{0}, n_{1}, \ldots, n_{m}\right)$-Hermite-Padé approximant $\left(P_{0}, P_{1}, \ldots, P_{m}\right)$ to the system

$$
\left(1-\lambda_{1} x\right)^{\alpha-n_{1}}, \ldots,\left(1-\lambda_{m} x\right)^{\alpha-n_{m}}
$$


is given by

$$
P_{0}(x)=p_{N}^{-\alpha}\left(0^{\left[n_{0}\right]},\left(-\lambda_{1} x\right)^{\left[n_{1}\right]}, \ldots,\left(-\lambda_{m} x\right)^{\left[n_{m}\right]}\right)
$$

and

$P_{j}(x)=\left(1-\lambda_{j} x\right)^{N-n_{j}} p_{N}^{-\alpha}\left(\left(\frac{\lambda_{j} x}{1-\lambda_{j} x}\right)^{\left[n_{0}\right]},\left(\frac{\left(\lambda_{j}-\lambda_{1}\right) x}{1-\lambda_{j} x}\right)^{\left[n_{1}\right]}, \ldots,\left(\frac{\left(\lambda_{j}-\lambda_{m}\right) x}{1-\lambda_{j} x}\right)^{\left[n_{m}\right]}\right)$,

with $N=\sum_{i=0}^{m} n_{i}$. Moreover, for $k=1, \ldots, m$, and for any $x$ such that $\left|\lambda_{j} x\right|<1$, we have

$$
\begin{aligned}
P_{0}(x)\left(1-\lambda_{j} x\right)^{\alpha-n_{j}}-P_{j}(x)=(-x)^{N+1} & \frac{(-N-\alpha-n)_{N+1}}{N !} \lambda_{j}{ }^{n_{0}+1}\left(1-\lambda_{j} x\right)^{\alpha-n_{j}} \\
& \times \int_{0}^{1}\left(1-\lambda_{j} x t\right)^{-\alpha-1} t^{n_{0}} \prod_{k=1}^{m}\left(t \lambda_{j}-\lambda_{k}\right)^{n_{j}} d t .
\end{aligned}
$$

Proof For any $j \in\{1, \ldots, m\}$, let us start again from (3.20), but now with

$$
\left(u_{1}, \ldots, u_{N}\right):=\left(0^{\left[n_{0}\right]},\left(-\lambda_{1} x\right)^{\left[n_{1}\right]}, \ldots,\left(-\lambda_{m} x\right)^{\left[n_{m}\right]}\right) .
$$

Following the same arguments as in the proof of Theorem the claimed properties are obtained by choosing $\beta:=-\alpha$.

Remark 3.4 The explicit expressions of the Hermite-Padé approximant obtained in the previous theorem are given below:

$$
P_{0}(x)=\sum_{k=0}^{N-n_{0}} \frac{(-N+\alpha)_{k}(-1)^{k}}{(-N)_{k}} \sigma_{k}\left(\lambda_{1}^{\left[n_{1}\right]}, \ldots, \lambda_{m}^{\left[n_{m}\right]}\right) x^{k}
$$

and for $j=1, \ldots, m$,

$$
\begin{aligned}
& P_{j}(x)= \\
& \quad \sum_{k=0}^{N-n} \frac{(-N+\alpha)_{k}}{(-N)_{k}} \sigma_{k}\left(\left(\lambda_{j}\right)^{\left[n_{0}\right]},\left(\lambda_{j}-\lambda_{1}\right)^{\left[n_{1}\right]}, \ldots,\left(\lambda_{j}-\lambda_{m}\right)^{\left[n_{m}\right]}\right) x^{k}\left(1-\lambda_{j} x\right)^{N-n_{j}-k} .
\end{aligned}
$$

\section{The $q$-world}

In the next section we will extend the results of Section 3 to the $q$-world, where from now on, $q<1$ is a given real number. It is therefore necessary to briefly review the main tools attached to it. 
4.1 $q$-calculus and $q$-hypergeometric series

We start with a brief survey on the main tools involved in the $q$-calculus. More details can be found in [18]. In the $q$-framework, the usual factorials are replaced by $q$ factorials defined by

$$
[0]_{q} !=1, \quad[k]_{q} !=[1]_{q}[2]_{q} \ldots[k]_{q}, \quad k \geq 1,
$$

where the symbols $[k]_{q}$, called $q$-integers, represent the quantities

$$
[k]_{q}:=1+q+\cdots+q^{k-1}=\frac{1-q^{k}}{1-q}, \quad k \geq 0 .
$$

Such $q$-factorials are involved in the expression of the $q$-binomial coefficients below:

$$
\left[\begin{array}{l}
n \\
k
\end{array}\right]_{q}=\frac{[n]_{q} !}{[k]_{q} ![n-k]_{q} !}, \quad 0 \leq k \leq n .
$$

For any real numbers $a$ and $b$, and any non-negative integer $n$, we use the notations $(a+b)_{q}^{n}$ and $(a-b)_{q}^{n}$ with the following meanings

$$
(a+b)_{q}^{n}:=(a+b)(a+q b) \ldots\left(a+q^{n-1} b\right), \quad(a-b)_{q}^{n}:=(a+(-b))_{q}^{n}
$$

As special cases, the $q$-Pochhammer symbols are defined by

$$
(b ; q)_{n}:=(1-b)_{q}^{n}=\prod_{k=0}^{n-1}\left(1-q^{k} b\right), \quad b \in \mathbb{R}, n \geq 0,
$$

with therefore $(b ; q)_{0}=1$. These symbols can even be extended to infinite products

$$
(b ; q)_{\infty}:=\prod_{k=0}^{+\infty}\left(1-q^{k} b\right) .
$$

Note that the $q$-Pochhammer symbols permit another expression of the $q$-binomial coefficients:

$$
\left[\begin{array}{l}
n \\
k
\end{array}\right]_{q}=\frac{(q ; q)_{n}}{(q ; q)_{k}(q ; q)_{n-k}}, \quad 0 \leq k \leq n .
$$

The $q$-derivative $D_{q}$ of a function $f$ is defined by

$$
D_{q} f(t)=\frac{f(q t)-f(t)}{(q-1) t}
$$

for all appropriate values of $t$. The $q$-derivative is a linear operator which satisfies the following product rule

$$
D_{q}(f g)(t)=D_{q} f(t) g(t)+f(q t) D_{q} g(t) .
$$

The definite $q$-integral of a function $f$ is defined by

$$
\int_{0}^{b} f(t) d_{q}(t)=(1-q) b \sum_{i=0}^{+\infty} q^{i} f\left(q^{i} b\right)=b \int_{0}^{1} f(b t) d_{q}(t)
$$


provided that the infinite sum converges. In case the function $f$ is continuous at 0 , then we automatically have

$$
f(b)-f(0)=\int_{0}^{b} D_{q} f(t) d_{q}(t)=b \int_{0}^{1} D_{q} f(b t) d_{q}(t) .
$$

With any given real numbers $a_{1}, \ldots, a_{r}, b_{1}, \ldots, b_{s}$, we can associate a $q$-hypergeometric function ${ }_{r} \phi_{s}\left(a_{1}, \ldots, a_{r} ; b_{1}, \ldots, b_{s} ; q,.\right)$ defined by $[13,14]$

$$
{ }_{r} \phi_{s}\left(a_{1}, \ldots, a_{r} ; b_{1}, \ldots, b_{s} ; q, x\right):=\sum_{k=0}^{+\infty} \frac{\left(a_{1} ; q\right)_{k}\left(a_{2} ; q\right)_{k} \ldots\left(a_{r} ; q\right)_{k}}{\left(b_{1} ; q\right)_{k}\left(b_{2} ; q\right)_{k} \ldots\left(b_{s} ; q\right)_{k}} \frac{x^{k}}{(q ; q)_{k}} .
$$

These series can be considered as $q$-generalisations of the ordinary hypergeometric function in so far as, when $q \rightarrow 1^{-}$, they give appropriate hypergeometric series. In case $b_{j}=q^{-K}$ for some $j \in\{1, \ldots, s\}$, where $K$ is a positive integer, the series is well defined only if one of the $a_{i}$ 's is equal to $q^{-L}$ for some positive integer $L \leq K$, again under the convention $0 / 0=0$. In next section, we will more specifically focus on the $q$-hypergeometric functions

$$
{ }_{1} \phi_{0}(\alpha ;-; q, x)=\sum_{k=0}^{+\infty} \frac{(\alpha ; q)_{k}}{(q ; q)_{k}} x^{k}, \quad|x|<1,
$$

where $\alpha$ is any non-zero real number. They are natural $q$-extensions of binomial functions since, for $|x|<1,(1-x)^{-\alpha}=\lim _{q \rightarrow 1^{-}} \phi_{0}(\alpha ;-; q, x)$, see [14]. We conclude this section by recalling the crucial binomial theorem which states that they can also be written as follows, [14]

$$
{ }_{1} \phi_{0}(\alpha ;-; q, x)=\frac{(\alpha x ; q)_{\infty}}{(x ; q)_{\infty}}, \quad|x|<1 .
$$

In particular it makes it makes it obvious that

$$
{ }_{1} \phi_{0}(\alpha ;-; q, x){ }_{1} \phi_{0}\left(\alpha^{-1} ;-; q, \alpha x\right)=1, \quad|x|,|\alpha x|<1 .
$$

\section{$4.2 q$-Blossoms: a brief survey and preliminary results}

The notion of $q$-blossoming was introduced in [29] as a generalisation of the classical notion of blossoming to the $q$-calculus framework. This generalisation is achieved by maintaining the symmetry and the multi-affinity of the classical notion while modifying the diagonal property. The precise definition is recalled below.

Definition 4.1 Let $P$ be a real polynomial of degree at most $N$. There exists a unique muti-affine symmetric function $\left(u_{1}, \ldots, u_{N}\right) \mapsto p\left(u_{1}, \ldots, u_{N}\right)$ which coincides with $P$ on the $q$-diagonal of $\mathbb{R}^{N}$, defined as the set of all $N$-tuples $\left(x, q x, \ldots, q^{N-1} x\right), x \in \mathbb{R}$. The function $p$ is called the $q$-blossom (in $N$ variables) of the polynomial $P$.

Remark 4.1 When $q$-blossoms were introduced in the initial paper [29], the notation used for them was $p\left(u_{1}, \ldots, u_{N} ; q\right)$. Since here, there will be no interference with ordinary blossoms, and since the number $q<1$ is fixed, we drop the $q$ for the sake of simplicity. 
When the polynomial $P$ is expressed in the monomial basis as $P(x)=\sum_{k=0}^{N} a_{k} x^{k}$, its $q$-blossom is explicitly given by

$$
p\left(u_{1}, u_{2}, \ldots, u_{N}\right)=\sum_{k=0}^{N} a_{k} \frac{\sigma_{k}\left(u_{1}, u_{2}, \ldots, u_{N}\right)}{\sigma_{k}\left(1, q, \ldots, q^{N-1}\right)},
$$

given that [29]

$$
\sigma_{k}\left(1, q, q^{2}, \ldots, q^{N-1}\right)=q^{k(k-1) / 2}\left[\begin{array}{l}
N \\
k
\end{array}\right]_{q}
$$

Example 4.1 As in the case of ordinary blossoms, it is not always necessary to use the expansion of a polynomial in the monomial basis to obtain its blossom. As an instance, given $a \in \mathbb{R}$, consider the degree $N$ polynomial $F_{N}(x):=(x+a)_{q}^{N}$. The $q$ blossom $f_{N}$ of $F_{N}$, in $N$ variables, can be expressed as follows:

$$
f_{N}\left(u_{1}, u_{2}, \ldots, u_{N}\right)=q^{N(N-1) / 2} \prod_{k=1}^{N}\left(u_{k} q^{-N+1}+a\right), \quad u_{1}, \ldots, u_{N} \in \mathbb{R} .
$$

Indeed, the function $f_{N}$ defined by (4.9) is clearly multi-affine and symmetric on $\mathbb{R}^{N}$. Moreover, on the $q$-diagonal, we have

$$
\begin{aligned}
f_{N}\left(x, q x, \ldots, q^{N-1} x\right) & =q^{N(N-1) / 2} \prod_{k=1}^{N}\left(x q^{-N+k}+a\right), \\
& =\prod_{k=1}^{N}\left[q^{N-k}\left(x q^{k-N}+a\right)\right]=(x+a)_{q}^{N}, \quad x \in \mathbb{R} .
\end{aligned}
$$

We now consider any two real numbers $a, b$, with $a \neq b$. In the $q$-world, we can go from the point $\left(a, q a, \ldots, q^{N-1} a\right)$ on the $q$-diagonal of $\mathbb{R}^{N}$ to the point $(b, q b, \ldots$, $q^{N-1} b$ ) by successively replacing $a$ by $b$, then $q a$ by $q b$, and so forth, up to $q^{N-1} a$ by $q^{N-1} b$. With any given polynomial $P$ of degree at most $N$, with $q$-blossom $p$, we thus associate the $(N+1)$ coefficients

$$
p_{k}=p\left(b, b q, \ldots, b q^{k-1}, a q^{k}, a q^{k+1}, \ldots, a q^{N-1}\right), \quad k=0, \ldots, N
$$

called the $q$-Bézier coefficients of $P$, relative to $(a, b)$. The multi-affinity and the symmetry of $q$-blossoms enable us to compute each value $p\left(u_{1}, \ldots, u_{N}\right)$ as an affine combination of the $q$-Bézier points $p_{0}, \ldots, p_{N}$, with coefficients independent of $P$, through a triangular $N$-step algorithm ( $q$-de Casteljau algorithm). This holds true in particular on the $q$-diagonal. We thus have, for all $x \in \mathbb{R}$,

$$
P(x)=p\left(x, q x, \ldots, q^{N-1} x\right)=\sum_{k=0}^{N} p_{k} B_{k}^{N}(x,(a, b) ; q), \quad \sum_{k=0}^{N} B_{k}^{N}(x,(a, b) ; q)=1,
$$

where the coefficients $B_{k}^{N}(x,(a, b) ; q), k=0, \ldots, N$, are independent of $P$. The $(N+1)$ functions $B_{k}^{N}(.,(a, b) ; q), k=0, \ldots, N$, thus form a basis of the degree $N$ polynomial 
space, called the $q$-Bernstein basis relative to $(a, b)$ [25,29,2]. A more detailed look at the $N$-step algorithm shows that, for $k=0, \ldots, N$,

$$
B_{k}^{N}(x,(a, b) ; q)=\left[\begin{array}{c}
N \\
k
\end{array}\right]_{q} \frac{(x-a)_{q}^{k}(b-x)_{q}^{N-k}}{(b-a)_{q}^{N}}, \quad x \in \mathbb{R} .
$$

We will need the following result.

Proposition 4.1 Let $n, N$ be two non-negative integers such that $n \leq N$, and let a be a real number. Then, given a polynomial $P$ of degree at most $N$, with $q$-blossom $p$ in $N$ variables, the following properties are equivalent:

(i) the polynomial $(.-a)_{q}^{n+1}$ divides $P$;

(ii) $p\left(u_{1}, \ldots, u_{n}, a q^{n}, a q^{n+1}, \ldots, a q^{N-1}\right)=0$ for all $u_{1}, \ldots, u_{n}$.

Proof Let linear space $\mathscr{E}$ of all $Q \in \mathbb{P}_{N}$ which are divisible by $(.-a)_{q}^{n+1}$ is $(N-$ $n$ )-dimensional. Clearly, given any real number $b \neq a$, the $q$-Bernstein functions $B_{j}^{N}(.,(a, b) ; q), j=n+1, \ldots, N$, form a basis of $\mathscr{E}$.

In other words, denoting by $p_{0}, \ldots, p_{N}$ the $q$-Bézier coefficients of $P$ relative to $(a, b)$, we can say that $P$ satisfies (i) if and only if $p_{0}=p_{1}=\cdots=p_{n}=0$. On the one hand, each among the $q$-Bézier coefficients $p_{0}, \ldots, p_{n}$ is of the form $p\left(u_{1}, \ldots, u_{n}, a q^{n}, a q^{n+1}, \ldots, a q^{N-1}\right)$ for some appropriate $u_{1}, \ldots, u_{n}$. On the other hand, for any $u_{1}, \ldots, u_{n} \in \mathbb{R}$, the symmetry and multi-affinity of $q$-blossoms enable us to obtain each $p\left(u_{1}, \ldots, u_{n}, a q^{n}, a q^{n+1}, \ldots, a q^{N-1}\right)=0$ as an affine combination of $p_{0}, \ldots, p_{n}$. These observations prove the equivalence between (i) and (ii).

Below we state the $q$-version of Proposition 2.2.

Proposition 4.2 Given $P \in \mathbb{P}_{N}$, with q-blossom $p$, expanded in the canonical basis as $P(x)=\sum_{k=0}^{N} a_{k} x^{k}$. Then, the polynomial function $\widetilde{P}$ of degree at most $(N-i)$ defined by

$$
\widetilde{P}(x):=p\left(0^{[i]}, q^{i} x, \ldots, q^{N-1} x\right), \quad x \in \mathbb{R}
$$

is expanded as

$$
\widetilde{P}(x)=\sum_{k=0}^{N-i} \widetilde{a}_{k} x^{k}, \quad \text { with } \widetilde{a}_{k}=a_{k} \frac{\left(q^{-N+i} ; q\right)_{k}}{\left(q^{-N} ; q\right)_{k}} \text { for } k=0, \ldots, N-i .
$$

Proof The integer $i \leq N$ being given, apply successively (4.7) and (4.8) to compute the quantity $\widetilde{P}(x)$. This gives

$$
\widetilde{P}(x)=\sum_{k=0}^{N-i} a_{k} \frac{\sigma_{k}\left(q^{i} x, \ldots, q^{N-1} x\right)}{\sigma_{k}\left(1, \ldots, q^{N-1}\right)}=\sum_{k=0}^{N-i} a_{k} b_{k} x^{k},
$$

with, due to (4.8) and to (4.1),

$$
\begin{aligned}
b_{k}: & =\frac{q^{k i} q^{k(k-1) / 2}\left[\begin{array}{c}
N-i \\
k
\end{array}\right]_{q}}{q^{k(k-1) / 2}\left[\begin{array}{c}
N \\
k
\end{array}\right]_{q}}=\frac{\left(q^{i}-q^{N-k+1}\right)\left(q^{i}-q^{N-k+2}\right) \ldots\left(q^{i}-q^{N}\right)}{\left(1-q^{N-k+1}\right)\left(1-q^{N-k+2}\right) \ldots\left(1-q^{N}\right)}, \\
& =\frac{\left(q^{-N+i+k-1}-1\right)\left(q^{-N+i+k-2}-1\right) \ldots\left(q^{-N+i}-1\right)}{\left(q^{-N+k-1}-1\right)\left(q^{-N+k-2}-1\right) \ldots\left(q^{-N}-1\right)}=\frac{\left(q^{-N+i} ; q\right)_{k}}{\left(q^{-N} ; q\right)_{k}},
\end{aligned}
$$

which proves the claim. 
Applied to $q$-hypergeometric series, this yields:

Corollary 4.1 Given any integers $r, s$, let $P_{N}$ be the degree $N$ truncation of the hypergeometric series ${ }_{r} \phi_{s}\left(a_{1}, \ldots, a_{r} ; b_{1}, \ldots, b_{s} ; q ;.\right)$, and let $p_{N}$ be its $q$-blossom (in $N$ variables). Then, for each $i=0, \ldots, N$,

$$
p_{N}\left(0^{[i]}, q^{i} x, \ldots, q^{N-1} x\right)={ }_{r+1} \phi_{s+1}\left(a_{1}, \ldots, a_{r}, q^{-N+i} ; b_{1}, \ldots, b_{s}, q^{-N} ; q ; x\right), \quad x \in \mathbb{R} .
$$

Observing that, when $x \neq 0, p_{N}\left(0^{[i]}, q^{i} x, \ldots, q^{N-1} x\right)$ is the $i$ th $q$-Bézier coefficient of $P_{N}$ relative to $(x, 0)$, the interesting following expansion readily follows.

Corollary 4.2 With the same notations as in Corollary 4.1, let $\beta$ be any non-zero real number. Then, the truncated function $P_{N}-$ which can also be described as $P_{N}=$ ${ }_{r+1} \phi_{s+1}\left(a_{1}, \ldots, a_{r}, q^{-N} ; b_{1}, \ldots, b_{s}, q^{-N} ; q ;.\right)$ - can be expanded in the q-Bernstein basis relative to $(\beta, 0)$ as follows:

$$
P_{N}=\sum_{i=0}^{N}{ }_{r+1} \phi_{s+1}\left(a_{1}, \ldots, a_{r},-N+i ; b_{1}, \ldots, b_{s},-N ; q ; \beta\right) B_{i}^{N}(.,(\beta, 0) ; q) .
$$

We conclude this section with the following result which will be useful in Subsection 3.4.

Corollary 4.3 Given any $a \in \mathbb{R}$, and any integer $\ell \leq N$, let $f_{\ell}$ denote the $q$-blossom in $N$ variables of the function $F_{\ell}(x):=(x+a)_{q}^{\ell}$. Then, there exist real numbers $A_{\ell, i}(q)$, independent of a, such that

$$
f_{\ell}\left(0^{[i]}, q^{i} a, \ldots, q^{N-1} a\right)=A_{\ell, i}(q) a^{\ell}, \quad i=0, \ldots, N .
$$

Proof Since $F_{\ell}(x)=(x+a)(x+q a) \ldots\left(x+q^{\ell-1} a\right)$, we have

$$
F_{\ell}(x)=\sum_{r=0}^{\ell} \sigma_{\ell-r}\left(1, q, \ldots, q^{\ell-1}\right) a^{\ell-r} x^{r}
$$

Accordingly, formula (4.10) yields the claimed result, with

$$
A_{\ell, i}(q)=\sum_{r=0}^{\min (\ell, N-i)} \sigma_{\ell-r}\left(1, q, \ldots, q^{\ell-1}\right) \frac{\left(q^{-N+i} ; q\right)_{r}}{\left(q^{-N} ; q\right)_{r}} .
$$

\section{Hermite identities and Padé approximants to $q$-hypergeometric functions}

In Section 3 we could observe that blossoms were elegant and efficient tools to obtain Padé approximants to some hypergeometric series. We now switch to the $q$ framework, where, as stated in the previous section, $q<1$ is a fixed real number. In this framework, $q$-blossoms will similarly provide us with Padé approximants to some $q$-hypergeometric series, through associated generalised Hermite identities. Though the general guiding line is the same as in Section 3, the proofs are sometimes significantly different. In addition to the specific difficulties of the $q$-calculus, this is due to the absence of something similar to the correspondence $\Theta$ introduced in Subsection 2.1 . 
5.1 Hermite identities for $q$-hypergeometric functions ${ }_{1} \phi_{0}(\alpha ;-; q ;$.

In this subsection we adapt the ideas developed in Subsection 3.1 to find a Hermite identity for the $q$-hypergeometric function ${ }_{1} \phi_{0}(\alpha ;-; q, x)$, where $\alpha$ is a given real number. With this in view, we first introduce the degree $N$ truncation of the expansion of ${ }_{1} \phi_{0}\left(q^{-N} \alpha ;-; q ;-.\right)$, that is, the polynomial

$$
P_{N}^{\alpha}(x):=\sum_{k=0}^{N} \frac{\left(q^{-N} \alpha ; q\right)_{k}}{(q ; q)_{k}}(-x)^{k}, \quad x \in \mathbb{R} .
$$

One major issue in this adaptation to the $q$-world was to find an appropriate bivariate polynomial $H_{N}^{\alpha}$ to work with. It turned out that we can use the following one:

$$
H_{N}^{\alpha}(x, u)=\sum_{k=0}^{N}(-1)^{k} \frac{\left(q^{-N} \alpha ; q\right)_{k}}{(q ; q)_{k}}(u+x)_{q}^{k}\left(1-\alpha q^{-1} x\right)_{q^{-1}}^{N-k}, \quad x \in \mathbb{R} .
$$

However, here, we do not have at our disposal a polynomial $Q_{N}^{\alpha}$ naturally connected with $P_{N}^{\alpha}$ via the bivariate polynomial $H_{N}^{\alpha}$ which, now, only interpolates $P_{N}^{\alpha}$ in the sense that

$$
H_{N}^{\alpha}(0, u)=P_{N}^{\alpha}(u), \quad u \in \mathbb{R} .
$$

The generalised Hermite identity for the $q$-hypergeometric function ${ }_{1} \phi_{0}(\alpha ;-; q, x)$ can be stated as follows.

Theorem 5.1 For any real numbers $\alpha$, any $u \in \mathbb{R}$, and any $x \in]-1,1[$, we have

$$
H_{N}^{\alpha}(x, u){ }_{1} \phi_{0}(\alpha ;-; q, x)-P_{N}^{\alpha}(u)=E_{N}^{\alpha}(x ; u),
$$

where $E_{N}^{\alpha}$ is given by

$$
E_{N}^{\alpha}(x, u)=(-1)^{N} x \frac{(1-\alpha)\left(q^{-N} \alpha ; q\right)_{N}}{(1-q)(q ; q)_{N}} \int_{0}^{1}{ }_{1} \phi_{0}(\alpha q ;-; q, t x)(u+q t x)_{q}^{N} d_{q} t
$$

To prove this, we will first establish the following lemma.

Lemma 5.1 For any real number $\alpha$, and any $u \in \mathbb{R}$ and any $x$ such that $|x|<1$, we have

$$
(1-\alpha x) D_{q} H_{N}^{\alpha}(., u)(x)+\frac{1-\alpha}{1-q} H_{N}^{\alpha}(q x, u)=(-1)^{N} \frac{(1-\alpha)\left(q^{-N} \alpha ; q\right)_{N}}{(1-q)(q ; q)_{N}}(u+q x)_{q}^{N}
$$

Proof The real number $u$ being given, for the sake of simplicity let us write the polynomial $H_{N}^{\alpha}(., u)$ as

$$
H_{N}^{\alpha}(., u)=\sum_{k=0}^{N} A_{k} f_{k} g_{N-k}\left(\alpha q^{-1} \cdot\right), \quad \text { with, for all } k \geq 0, A_{k}:=(-1)^{k} \frac{\left(q^{-N} \alpha ; q\right)_{k}}{(q ; q)_{k}}
$$


and where the functions $f_{k}, g_{k}$ are defined by

$$
f_{k}(x):=(u+x)_{q}^{k}, \quad g_{k}(x):=(1-x)_{q^{-1}}^{k} .
$$

It is easily checked that, for $k \geq 0$,

$$
D_{q} f_{k}(x)=[k]_{q} f_{k-1}(q x), \quad D_{q} g_{k}(x)=-[k]_{q^{-1}} g_{k-1}(x),
$$

in which the functions $f_{-1}, g_{-1}$ are identically zero. Using the differentiation formula (4.2) we thus obtain,

$$
\begin{gathered}
D_{q}\left[f_{k} g_{N-k}\left(\alpha q^{-1} \cdot\right)\right](x)=D_{q} f_{k}(x) g_{N-k}\left(\alpha q^{-1} x\right)+f_{k}(q x) \alpha q^{-1} D_{q} g_{N-k}\left(\alpha q^{-1} x\right), \\
=[k]_{q} f_{k-1}(q x) g_{N-k}\left(\alpha q^{-1} x\right)-\alpha q^{-1} f_{k}(q x)[N-k]_{q^{-1}} g_{N-k-1}\left(\alpha q^{-1} x\right) .
\end{gathered}
$$

Accordingly,

$$
\begin{aligned}
& D_{q} H_{N}^{\alpha}(., u)(x)= \\
& \quad \sum_{k=0}^{N-1}\left[A_{k+1}[k+1]_{q}-\alpha q^{-1} A_{k}[N-k]_{q^{-1}}\right] g_{N-k-1}\left(\alpha q^{-1} x\right) f_{k}(q x) .
\end{aligned}
$$

Given that

$$
(1-\alpha x) g_{N-k-1}\left(\alpha q^{-1} x\right)=g_{N-k}(\alpha x), \quad A_{k+1}=-A_{k} \frac{1-\alpha q^{-N+k}}{1-q^{k+1}},
$$

after multiplication by $(1-\alpha x)$, the identity (5.6) eventually reduces to

$$
\begin{aligned}
(1-\alpha x) D_{q} H_{N}^{\alpha}(., u)(x) & =\frac{\alpha-1}{1-q} \sum_{k=0}^{N-1} A_{k} f_{k}(q x) g_{N-k}(\alpha x), \\
& =\frac{\alpha-1}{1-q}\left[H_{N}^{\alpha}(q x, u)-A_{N} f_{N}(q x)\right]
\end{aligned}
$$

This is exactly the claim (5.4).

Proof of Theorem 5.1. For any given $u \in \mathbb{R}$, we can apply the integration formula (4.3) to the function $H_{N}^{\alpha}(., u)_{1} \phi_{0}(\alpha ;-; q,$.$) which is continuous at the origin. Ac-$ cordingly, taking account of (5.1), to obtain the claimed relations (5.2) and (5.3) it is sufficient to prove that

$$
\begin{aligned}
& D_{q}\left[H_{N}^{\alpha}(., u){ }_{1} \phi_{0}(\alpha ;-; q, .)\right](x)= \\
& \left.\quad(-1)^{N} \frac{(1-\alpha)\left(q^{-N} \alpha ; q\right)_{N}}{(1-q)(q ; q)_{N}}{ }_{1} \phi_{0}(\alpha q ;-; q, x)(u+q x)_{q}^{N}, \quad x \in\right]-1,1[.
\end{aligned}
$$

Let us thus compute the $q$-derivative of the product $\left[H_{N}^{\alpha}(., u){ }_{1} \phi_{0}(\alpha ;-, q,).\right]$. On account of the differentiation formula (4.2), we have, for $|x|<1$,

$$
\begin{aligned}
D_{q}\left[H_{N}^{\alpha}(., u){ }_{1} \phi_{0}(\alpha ;-; q, .)\right](x)={ }_{1} \phi_{0}(\alpha ;-; q, x) D_{q} H_{N}^{\alpha}(., u)(x) \\
+H_{N}^{\alpha}(q x, u) D_{q}\left({ }_{1} \phi_{0}(\alpha ;-; q, .)\right)(x) .
\end{aligned}
$$


Using the $q$-binomial relation (4.4), it is easily checked that

$$
D_{q}\left({ }_{1} \phi_{0}(\alpha ;-; q, .)\right)(x)=\frac{1-\alpha}{1-q} \frac{{ }_{1} \phi_{0}(\alpha ;-; q, x)}{(1-\alpha x)}=\frac{1-\alpha}{1-q}{ }_{1} \phi_{0}(\alpha q ;-; q, x) .
$$

Substituting this relation in (5.8) leads to

$$
\begin{aligned}
& D_{q}\left[H_{N}^{\alpha}(., u){ }_{1} \phi_{0}(\alpha ;-; q, .)\right](x) \\
& \quad={ }_{1} \phi_{0}(\alpha q ;-; q, x)\left[(1-\alpha x) D_{q} H_{N}^{\alpha}(., u)(x)+\frac{1-\alpha}{1-q} H_{N}^{\alpha}(q x, u)\right] .
\end{aligned}
$$

Due to Lemma 5.1, the proof of (5.7) is complete.

Subsequently we denote by $p_{N}^{\alpha}$, and for any given $x \in \mathbb{R}$ by $h_{N}^{\alpha}(x ;$.$) the q$-blossoms of $P_{N}^{\alpha}$ and $H_{N}^{\alpha}(x ; \bullet)$, respectively, both defined on $\mathbb{R}^{N}$. The true $q$-Hermite identity on which the construction of Padé approximants will rely is the $q$-blossomed version of Theorem 5.1, that is:

Theorem 5.2 For any real numbers $\alpha, u_{1}, u_{2}, \ldots, u_{N}$, and any $\left.x \in\right]-1,1[$, we have

$$
h_{N}^{\alpha}\left(x ; u_{1}, u_{2}, \ldots, u_{N}\right){ }_{1} \phi_{0}(\alpha ;-, q, x)-p_{N}^{\alpha}\left(u_{1}, u_{2}, \ldots, u_{N}\right)=e_{N}^{\alpha}\left(x ; u_{1}, u_{2}, \ldots, u_{N}\right),
$$

where $e_{N}^{\alpha}$ is given by

$$
\begin{array}{rl}
e_{N}^{\alpha}\left(x ; u_{1}, u_{2}, \ldots, u_{N}\right)=(-1)^{N} & x \frac{(1-\alpha)\left(q^{-N} \alpha ; q\right)_{N}}{(1-q)(q ; q)_{N}} q^{N(N-1) / 2} \\
& \times \int_{0}^{1}{ }_{1} \phi_{0}(\alpha q ;-; q, t x) \prod_{i=1}^{N}\left(q^{-N+1} u_{i}+q t x\right) d_{q} t .
\end{array}
$$

Proof For each $x \in]-1,1$ [, both sides of (5.2) are polynomials of degree at most $N$ in the variable $u$. Since they coincide on $\mathbb{R}$, so do their $q$-blossoms on $\mathbb{R}^{N}$, which yields (5.9). Indeed, taking account of formula (4.9) in Example 4.1, one can see that the function $e_{N}^{\alpha}(x ;$.$) defined in (5.10) is the q$-blossom of the function $E_{N}^{\alpha}(x ;$.$) defined$ in (5.3).

5.2 Padé approximants to the hypergeometric function ${ }_{1} \phi_{0}(\alpha ;-, q,$.

The purpose of this subsection is to produce Padé approximants to a given hypergeometric function ${ }_{1} \phi_{0}(\alpha ;-; q,$.$) . We will more precisely establish the following result.$

Theorem 5.3 Given any non-negative integers $m, n$ and any $\alpha \in \mathbb{R}$, an $[m / n]$-Padé approximant $\left(P_{m}, Q_{n}\right)$ to the q-hypergeometric function ${ }_{1} \phi_{0}(\alpha ;-, q,$.$) is given by$

$$
\left(P_{m}, Q_{n}\right)=\left[{ }_{2} \phi_{1}\left(q^{-n} \alpha, q^{-m} ; q^{-N} ; q,-.\right),{ }_{2} \phi_{1}\left(q^{-m} \alpha^{-1}, q^{-n} ; q^{-N} ; q,-\alpha .\right)\right],
$$

with $N:=m+n$. Moreover, for $|x|<1$, the remainder

$$
R_{m, n}(x):=Q_{n}(x){ }_{1} \phi_{0}(\alpha ;-, q, x)-P_{m}(x)
$$


is given by

$$
\begin{aligned}
& R_{m, n}(x)=(-1)^{N} x^{N+1} \frac{\left(1-\alpha q^{m}\right)\left(q^{-n} \alpha ; q\right)_{N}}{(1-q)(q ; q)_{N}} q^{N(N+1) / 2} \\
& \times \int_{0}^{1}{ }_{1} \phi_{0}\left(q^{m+1} \alpha ;-; q, x t\right) t^{n}\left(t-q^{-m}\right)_{q}^{m} d_{q} t .
\end{aligned}
$$

Proof Let $\beta$ be a real number. For $|x|<1$, apply the associated generalised Hermite identity (5.9) to the $N$-tuple

$$
\begin{gathered}
\left(u_{1}, \ldots, u_{N}\right):=\left(0^{[n]}, X_{q}(x)\right), \quad \text { with } X_{q}(x):=\left(-q^{n} x,-q^{n+1} x, \ldots,-q^{N-1} x\right) . \\
h_{N}^{\beta}\left(x ; 0^{[n]}, X_{q}(x)\right){ }_{1} \phi_{0}(\beta ;-; q, x)-p_{N}^{\beta}\left(0^{[n]}, X_{q}(x)\right)=R_{N}(x)
\end{gathered}
$$

where, according to (5.10)

$$
\begin{aligned}
& R_{N}(x)=(-1)^{N} x^{N+1} \frac{(1-\beta)\left(q^{-N} \beta ; q\right)_{N}}{(1-q)(q ; q)_{N}} q^{N(N+1) / 2} \\
& \quad \times \int_{0}^{1}{ }_{1} \phi_{0}(q \beta ;-; q, x t) t^{n}\left(t-q^{-m}\right)_{q}^{m} d_{q} t .
\end{aligned}
$$

According to Proposition 4.1, on any $N$-tuple of the form $\left(v_{1}, \ldots, v_{n}, X_{q}(x)\right)$, the $q$ blossom $h_{N}^{\beta}(x ;$.$) of the polynomial H_{N}^{\beta}(x,$.$) coincides with the q$-blossom (in $N$ variables) of its degree $n$ truncation $H_{N, n}^{\beta}(x,$.$) , that is$

$$
\begin{aligned}
H_{N, n}^{\beta}(x, u) & =\sum_{k=0}^{n}(-1)^{k} \frac{\left(q^{-N} \beta ; q\right)_{k}}{(q ; q)_{k}}(u+x)_{q}^{k}\left(1-\beta q^{-1} x\right)_{q^{-1}}^{N-k} \\
& =\left(1-\beta q^{-1} x\right)_{q^{-1}}^{m} G_{N, n}^{\beta}(x, u),
\end{aligned}
$$

where the bivariate polynomial $G_{N, n}^{\beta}$ is defined by

$$
G_{N, n}^{\beta}(x, u)=\sum_{k=0}^{n}(-1)^{k} \frac{\left(q^{-N} \beta ; q\right)_{k}}{(q ; q)_{k}}(u+x)_{q}^{k}\left(1-q^{-m-1} \beta x\right)_{q^{-1}}^{n-k} .
$$

In other words, if $g_{N, n}^{\beta}(x ; \bullet)$ is the blossom in $N$ variables of the polynomial $G_{N, n}^{\beta}(x, \bullet)$, we have, for all $v_{1}, \ldots, v_{n}$,

$$
h_{N}^{\beta}\left(x ; v_{1}, \ldots, v_{n}, X_{q}(x)\right)=\left(1-\beta q^{-1} x\right)_{q^{-1}}^{m} g_{N, n}^{\beta}\left(x ; v_{1}, \ldots, v_{n}, X_{q}(x)\right) .
$$

Thus, in particular, (5.13) can be written as follows:

$$
\left(1-\beta q^{-1} x\right)_{q^{-1}}^{m} g_{N, n}^{\beta}\left(x ; 0^{[n]}, X_{q}(x)\right){ }_{1} \phi_{0}(\beta ;-; q, x)-p_{N}^{\beta}\left(0^{[n]}, X_{q}(x)\right)=R_{N}(x) .
$$

The $q$-binomial theorem (4.4) ensures that

$$
\left(1-\beta q^{-1} x\right)_{q^{-1}{ }_{1}}^{m} \phi_{0}(\beta ;-; q, x)={ }_{1} \phi_{0}\left(q^{-m} \beta ;-; q, x\right) .
$$


Therefore, (5.13) is eventually transformed into:

$$
g_{N, n}^{\beta}\left(x ; 0^{[n]}, X_{q}(x)\right){ }_{1} \phi_{0}\left(q^{-m} \beta ;-; q, x\right)-p_{N}^{\beta}\left(0^{[n]}, X_{q}(x)\right)=R_{N}(x) .
$$

The function $x \in \mathbb{R} \mapsto p_{N}^{\beta}\left(0^{[n]}, X_{q}(x)\right)$ is clearly a polynomial of degree at most $m$ in $x$. As for the function $x \in \mathbb{R} \mapsto g_{N, n}^{\beta}\left(x ; 0^{[n]}, X_{q}(x)\right)$, we are actually interested in proving that it is of degree at most $n$. Now, applying Corollary 4.3 to each $(.+x)_{q}^{k}$, $k=0, \ldots, n$, we can state that

$$
g_{N, n}^{\beta}\left(x ; 0^{[n]}, X_{q}(x)\right)=\sum_{k=0}^{n} A_{k, n}(q) x^{k}\left(1-q^{-m-1} \beta x\right)_{q^{-1}}^{n-k} .
$$

the real numbers $A_{k, n}(q)$ being those defined in (4.12). This is indeed a polynomial of degree at most $n$ in $x$.

Take $\beta=\alpha q^{m}$. Then, (5.14) yields

$$
g_{N, n}^{\alpha q^{m}}\left(x ; 0^{[n]}, X_{q}(x)\right){ }_{1} \phi_{0}(\alpha ;-; q, x)-p_{N}^{\alpha q^{m}}\left(0^{[n]}, X_{q}(x)\right)=R_{m, n}(x),
$$

where the quantity $R_{m, n}(x)$ is given in (5.12). Therefore, at this stage, we can state the intermediate result:

Proposition 5.1 For any $\alpha \in \mathbb{R}$, an $[\mathrm{m} / n]$-Padé approximant $\left(P_{m}, Q_{n}\right)$ to the hypergeometric function ${ }_{1} \phi_{0}(\alpha ;-; q,$.$) is given by$

$$
\begin{aligned}
& P_{m}(x)=p_{N}^{\alpha q^{m}}\left(0^{[n]},-q^{n} x, \ldots,-q^{N-1} x\right), \\
& Q_{n}(x)=g_{N, n}^{\alpha q^{m}}\left(x ; 0^{[n]},-q^{n} x, \ldots,-q^{N-1} x\right), \quad x \in \mathbb{R} .
\end{aligned}
$$

End of the proof of Theorem 5.3: Formulæ (5.15) already provide us with an explicit expression of the $[m / n]$-Padé approximant $\left(P_{m}, Q_{n}\right)$ to the function ${ }_{1} \phi_{0}(\alpha ;-, q,$.$) . It$ remains to show that this coincides with the announced relation (5.11). That

$$
P_{m}(x)={ }_{2} \phi_{1}\left(q^{-n} \alpha, q^{-m} ; q^{-N} ; q,-x\right), \quad x \in \mathbb{R},
$$

readily follows by applying Corollary 4.1 to ${ }_{1} \phi_{0}\left(q^{-N} \alpha ;-; q ;.\right)$. To complete the proof of (5.11), observe that $\left(Q_{n}, P_{m}\right)$ is an $[n / m]$-Padé approximant to the inverse of ${ }_{1} \phi_{0}(\alpha ;-, q,$.$) (see Subsection 2.2). From the q$-binomial theorem (4.4), we know that

$$
{ }_{1} \phi_{0}(\alpha ;-; q, y){ }_{1} \phi_{0}\left(\alpha^{-1} ;-; q, \alpha y\right)=1, \quad|y|,|\alpha y|<1 .
$$

Accordingly, we can say that $\left(Q_{n}(\bullet / \alpha), P_{m}(\bullet / \alpha)\right)$ is an $[n / m]$-Padé approximant to the $q$-hypergeometric function ${ }_{1} \phi_{0}\left(\alpha^{-1} ;-; q,.\right)$.

Suppose for a while that $\left(P_{m}, Q_{n}\right)$ is the only $[m / n]$-Padé approximant to the $q$-hypergeometric function ${ }_{1} \phi_{0}(\alpha ;-, q,$.$) . Then, \left(Q_{n}(\cdot / \alpha), P_{m}(\cdot / \alpha)\right)$ is the only $[n / m]$-Padé approximant to ${ }_{1} \phi_{0}\left(\alpha^{-1} ;-; q,.\right)$. From $(5.16)$ we can conclude that

$$
Q_{n}(x / \alpha)={ }_{2} \phi_{1}\left(q^{-m} \alpha^{-1}, q^{-n} ; q^{-N} ; q,-x\right), \quad x \in \mathbb{R},
$$


which is precisely the right part of (5.11). Now, the function ${ }_{1} \phi_{0}(\alpha ;-, q,$.$) possesses$ a unique $[m / n]$-Padé approximant if and only if the determinant $\Delta_{m, n}\left({ }_{1} \phi_{0}(\alpha ;-, q, \bullet)\right)$, defined according to (2.11), is not zero. This determinant being a polynomial in the variable $\alpha$, so far we can state that the right part of (5.11) is proved for all $\alpha \in \mathbb{R}$, save for a finite number of values. For any $x \in \mathbb{R}$, both $\alpha \mapsto Q_{n}(x)$ and $\alpha \mapsto{ }_{2} \phi_{1}\left(q^{-m} \alpha^{-1}, q^{-n} ; q^{-N} ; q,-\alpha x\right)$ being continuous functions, we can conclude that (5.11) is valid for any value of $\alpha$.

\subsection{Type II Hermite-Padé Approximation}

Based on the Hermite identity (5.9), we shall now provide explicit expressions of the $\left(n_{0}, n, \ldots, n\right)$-Hermite-Padé approximation to the system

$$
{ }_{1} \phi_{0}\left(\alpha ;-; q, \lambda_{1} x\right), \ldots,{ }_{1} \phi_{0}\left(\alpha ;-; q, \lambda_{m} x\right)
$$

where $\lambda_{1}, \ldots, \lambda_{m}$ are given pairwise distinct non-zero real numbers, and $n_{0}, n$ are any non-negative integers.

Let us set $N:=n_{0}+m n$. Take any arbitrary pairwise distinct non-zero real numbers $\mu_{1}, \ldots, \mu_{m}$, and consider the $N$-tuple

$$
\left(u_{1}, \ldots, u_{N}\right)=\left(U_{0}(x), \ldots, U_{m}(x)\right) \text {, }
$$

with $U_{0}(x)=\left(0^{\left[n_{0}\right]}\right)$ and

$$
U_{j}(x)=\left(-q^{N-n} \mu_{j} x,-q^{N-n+1} \mu_{j} x, \ldots,-q^{N-1} \mu_{j} x\right) \text { for } j=1, \ldots, m .
$$

Given any real number $\beta$, and given an integer $k \in\{1, \ldots, n\}$, the Hermite identity (5.9) shows that, for $\left|\mu_{k} x\right|<1$, the quantity

$$
h_{N}^{\beta}\left(\mu_{k} x ; U_{0}(x), \ldots, U_{m}(x)\right){ }_{1} \phi_{0}\left(\beta ;-; q, \mu_{k} x\right)-p_{N}^{\beta}\left(U_{0}(x), \ldots, U_{m}(x)\right)
$$

is equal to $R_{N}^{k}(x)$, where

$$
\begin{aligned}
& R_{N}^{k}(x)=(-1)^{N} x^{N+1} \mu_{k}^{n_{0}+1} \frac{(1-\beta)\left(q^{-N} \beta ; q\right)_{N}}{(1-q)(q ; q)_{N}} q^{N(N+1) / 2} \\
& \quad \times \int_{0}^{1}{ }_{1} \phi_{0}\left(q \beta ;-; q, \mu_{k} x t\right) t^{n_{0}} \prod_{j=1}^{m}\left(t \mu_{k}-q^{-n} \mu_{j}\right)_{q}^{n} d_{q} t .
\end{aligned}
$$

Since $U_{0}(x)=\left(0^{\left[n_{0}\right]}\right)$, the function $x \mapsto p_{N}^{\beta}\left(U_{0}(x), U_{1}(x), \ldots, U_{m}(x)\right)$ is clearly a polynomial of degree at most $N-n_{0}$. Due to Proposition 4.1, the presence of $U_{k}(x)$ in the $N$-tuple $\left(U_{0}(x), \ldots, U_{m}(x)\right)$ guarantees that we can skip all terms $\left(.+\mu_{k} x\right)_{q}^{k}$ with $k>N-n$ when calculating $h_{N}^{\beta}\left(\mu_{k} x ; U_{0}(x), U_{1}(x), \ldots, U_{m}(x)\right)$. Following arguments similar to those used in the proof of Theorem 5.3, let $G_{N, N-n}^{\beta}\left(\mu_{k} x ;.\right)$ denote 
the polynomial obtained by dividing the degree $(N-n)$ truncation of the polynomial $H_{N}^{\beta}\left(\mu_{k} x ;\right.$. ) by $\left(1-\beta \mu_{k} q^{-1} x\right)_{q^{-1}}^{n}$. We similarly obtain, for $\left|\mu_{k} x\right|<1$,

$$
\begin{array}{r}
g_{N, N-n}^{\beta}\left(\mu_{k} x ; U_{0}(x), \ldots, U_{m}(x)\right){ }_{1} \phi_{0}\left(q^{-n} \beta ;-; q, \mu_{k} x\right) \\
-p_{N}^{\beta}\left(U_{0}(x), \ldots, U_{m}(x)\right)=R_{N}^{k}(x),
\end{array}
$$

where $g_{N, N-n}^{\beta}\left(\mu_{k} x ;\right.$. $)$ is the $q$-blossom in $N$ variables of $G_{N, N-n}^{\beta}\left(\mu_{k} x ;.\right)$. Moreover, as in the proof of Theorem 5.3, from Corollary 4.3 we can derive that the function $x \mapsto g_{N-n}^{\beta}\left(\mu_{k} x ; U_{0}(x), \ldots, U_{m}(x)\right)$ is a polynomial of degree at most $N-n$.

Additionally assuming that $\left|q^{-n} \beta \mu_{k} x\right|<1$, multiply both hand-sides of the previous equality by $-{ }_{1} \phi_{0}\left(q^{n} \beta^{-1} ;-; q, q^{-n} \beta \mu_{k} x\right)$. This yields:

$$
\begin{aligned}
& p_{N}^{\beta}\left(U_{0}(x), \ldots, U_{m}(x)\right){ }_{1} \phi_{0}\left(q^{n} \beta^{-1} ;-; q, q^{-n} \beta \mu_{k} x\right)- \\
& \quad g_{N, N-n}^{\beta}\left(\mu_{k} x ; U_{0}(x), \ldots, U_{m}(x)\right)=-R_{N}^{k}(x){ }_{1} \phi_{0}\left(q^{n} \beta^{-1} ;-; q, q^{-n} \beta \mu_{k} x\right) .
\end{aligned}
$$

Applying these relations with $\beta=\alpha^{-1} q^{n}$ and $\mu_{j}=\alpha \lambda_{j}, j=1, \ldots, m$, this eventually provides us with the result below.

Theorem 5.4 Given non-negative integers $n_{0}, n$, an $\left(n_{0}, n, \ldots, n\right)$-Hermite-Padé approximant $\left(P_{0}, P_{1}, \ldots, P_{m}\right)$ to the system (5.17) is given by

$$
\begin{aligned}
& P_{0}(x)=p_{N}^{\alpha^{-1} q^{n}}\left(0^{\left[n_{0}\right]}, V_{1}(x), \ldots, V_{m}(x)\right) \\
& P_{k}(x)=g_{N-n}^{\alpha^{-1} q^{n}}\left(\alpha \lambda_{k} x ; 0^{\left[n_{0}\right]}, V_{1}(x) \ldots, V_{m}(x)\right), \quad k=1, \ldots, m,
\end{aligned}
$$

where

$$
V_{j}(x)=\left(-q^{N-n} \alpha \lambda_{j} x,-q^{N-n+1} \alpha \lambda_{j} x, \ldots,-q^{N-1} \alpha \lambda_{j} x\right), \quad j=1, \ldots, m .
$$

Moreover, for $k=1,2, \ldots, m$ and for $\left|\lambda_{k} x\right|<\min \left(1, \frac{1}{\alpha}\right)$, the remainder

$$
R_{N}^{k}(x):=P_{0}(x){ }_{1} \phi_{0}\left(\alpha ;-; q, \lambda_{k} x\right)-P_{k}(x)
$$

can be expressed as

$$
\begin{aligned}
R_{N}^{k}(x)=(- & \alpha x)^{N+1} \lambda_{k}{ }^{n_{0}+1} \frac{\left(1-q^{n} \alpha^{-1}\right)\left(q^{-N+n} \alpha^{-1} ; q\right)_{N}}{(1-q)(q ; q)_{N}}{ }_{1} \phi_{0}\left(\alpha ;-; q, \lambda_{k} x\right) \\
& \times q^{N(N+1) / 2} \int_{0}^{1}{ }_{1} \phi_{0}\left(q^{n+1} \alpha^{-1} ;-; q, \alpha \lambda_{k} x t\right) t^{n_{0}} \prod_{j=1}^{m}\left(t \lambda_{k}-q^{-n} \lambda_{j}\right)_{q}^{n} d_{q} t .
\end{aligned}
$$

Remark 5.1 The polynomials $G_{N, N-n}^{\beta}(x,),. p_{N}^{\beta}$ are linear combinations of functions of the form $F_{\ell}(u)=(u+\lambda x)_{q}^{\ell}$, for some $\ell \leq N-n$ and some real number $\lambda$. More explicit expressions for the polynomials $P_{k}, k=0,1, \ldots, m$, are thus obtained by $q$ blossoming in $N$ variables the expansion in the monomial basis of the function $F_{\ell}$ as 
was already done in the proof of Corollary 4.3, but with now $a=\lambda x$. Denoting by $f_{\ell}$ this $q$-blossom, it is easily checked that

$$
f_{\ell}\left(0^{\left[n_{0}\right]}, V_{1}(x), \ldots, V_{m}(x)\right)=x^{\ell} \sum_{r=0}^{\min (\ell, N-n)} \frac{\left[\begin{array}{l}
\ell \\
r
\end{array}\right]_{q}}{\left[\begin{array}{l}
N \\
r
\end{array}\right]_{q}} q^{\ell^{2}-(2 r+1) \ell / 2} \lambda^{\ell-r} \sigma_{r}\left(\widetilde{V}_{1}, \ldots, \widetilde{V}_{m}\right),
$$

where $\widetilde{V}_{j}:=\left(q^{N-n} \alpha \lambda_{j}, q^{N-n+1} \alpha \lambda_{j}, \ldots, q^{N-1} \alpha \lambda_{j}\right)$ for $j=1, \ldots, m$.

We conclude this section with $q$-version of Theorem 3.5.

Theorem 5.5 Given any sequence $\left(n_{0}, \ldots, n_{m}\right)$ of non-negative integers, and any real number $\alpha$, the $\left(n_{0}, \ldots, n_{m}\right)$-Hermite-Padé approximant $\left(P_{0}, P_{1}, \ldots, P_{m}\right)$ to the system

$$
{ }_{1} \phi_{0}\left(q^{n_{1}} \alpha ;-; q, \lambda_{1} x\right), \ldots,{ }_{1} \phi_{0}\left(q^{n_{m}} \alpha ;-; q, \lambda_{m} x\right),
$$

is given by

$$
\begin{aligned}
& P_{0}(x)=p_{N}^{\alpha^{-1}}\left(0^{\left[n_{0}\right]}, V_{1}(x), \ldots, V_{m}(x)\right), \\
& P_{k}(x)=g_{N-n_{k}}^{\alpha^{-1}}\left(\alpha \lambda_{k} x ; 0^{\left[n_{0}\right]}, V_{1}(x), \ldots, V_{m}(x)\right), \quad k=1, \ldots, m,
\end{aligned}
$$

where $N=\sum_{i=0}^{n} n_{i}$ and, for $j=1, \ldots, m$,

$$
V_{j}(x)=\left(-q^{N-n_{j}} \alpha \lambda_{j} x,-q^{N-n_{j}+1} \alpha \lambda_{j} x, \ldots,-q^{N-1} \alpha \lambda_{j} x\right)
$$

Moreover, for $k=1,2, \ldots, m$ and for $\left|\lambda_{k} x\right|<\min \left(1, \frac{1}{\alpha}\right)$, the remainder

$$
R_{N}^{k}(x):=P_{0}(x){ }_{1} \phi_{0}\left(q^{n_{k}} \alpha ;-; q, \lambda_{k} x\right)-P_{k}(x)
$$

can be expressed as

$$
\begin{aligned}
R_{N}^{k}(x)=(- & \alpha x)^{N+1} \lambda_{k}{ }^{n_{0}+1} \frac{1-\alpha^{-1}}{1-q} \frac{\left(q^{-N} \alpha^{-1} ; q\right)_{N}}{(q ; q)_{N}}{ }_{1} \phi_{0}\left(q^{n_{k}} \alpha ;-; q, \lambda_{k} x\right) \\
& \times q^{N(N+1) / 2} \int_{0}^{1}{ }_{1} \phi_{0}\left(\alpha^{-1} q ;-; q, \alpha \lambda_{k} x t\right) t^{n_{0}} \prod_{j=1}^{m}\left(t \lambda_{k}-q^{-n_{j}} \lambda_{j}\right)_{q}^{n_{j}} d_{q} t
\end{aligned}
$$

Proof Apply the same technique as in the proof of Theorem 5.4 to calculate (5.19) and the corresponding remainders, with now

$$
U_{j}(x)=\left(-q^{N-n_{j}} \mu_{j} x,-q^{N-n_{j}+1} \mu_{j} x, \ldots,-q^{N-1} \mu_{j} x\right), \quad j=1, \ldots, m
$$

In the end we have to choose $\beta:=\alpha^{-1}$ and $\mu_{k}:=\alpha \lambda_{k}$. 


\section{Conclusion}

This work has demonstrated the efficiency of blossoms to produce Padé approximants to certain functions $\psi$. The initial key idea of the corresponding strategy consists, if it is possible, in associating with $\psi$ a Hermite identity of the form

$$
H(x, u) \psi(x)-H(0, u)=R(x, u)
$$

where for each convenient $x, H(x, u)$ and $R(x, u)$ are appropriate polynomial expressions in the variable $u$. The second one consists in applying the blossomed version of this identity to relevant tuples so as to simultaneously generate Hermite-Padé approximants and their remainders.

The underlying reason why we were able to find Hermite identities of the form (6.1) for the functions $\psi$ we considered, is the fact that these functions satisfy a first order linear differential equation ( $q$-difference equation in the quantum context) with polynomial coefficients of appropriate degrees. Beyond this simple case, if the differential equation is of higher order, we will have to determine a more complicated Hermite identity, involving not only the function but also its derivatives, [17]. In that case, our future strategy will consist in inserting specific arguments in the blossoms to eliminate the derivatives, so as to then be able to produce Padé and type II HermitePadé approximants.

\section{References}

1. R. Ait-Haddou, q-Blossoming and Hermite-Padé approximants to the q-exponential function, Numer. Algorithms, 76 (2017), 53-66.

2. R. Ait-Haddou, R. Goldman, Best polynomial degree reduction on $q$-lattices with applications to $q$ orthogonal polynomials. App. Math Comp., 266 (2015), 267-276.

3. R. Ait-Haddou, M.-L. Mazure, The Fundamental Blossoming Inequality in Chebyshev Spaces I: Applications to Schur Functions. Found. Comp. Math. 18 (2018), 135-158.

4. A. Baker, Rational approximations to $\sqrt[3]{2}$ and other algebraic numbers, Quart. J. Math., 16 (1964), 375-383; Matematika, 9 (1965), 128-135.

5. G.A. Baker, Essentials of Padé Approximants, Academic Press, New York, 1975.

6. P.B. Borwein, Padé approximants for the q-elementary functions, Constr. Approx., 4 (1988), 391-402.

7. C. Brezinski, H. Padé ; Oeuvres, Albert Blanchard, Paris, 1984

8. M.G. de Bruin, Some convergence results in simultaneous rational approximation to the set of hypergeometric functions $\left\{{ }_{1} F_{1}\left(1 ; c_{i} ; z\right)\right\}_{i=1}^{n}$, in Padé Approximation and its Applications, Lecture Notes in Mathematics, 1071, 12-33, Springer, Berlin, 1984.

9. M.G. de Bruin, Simultaneous rational approximation to some $q$-hypergeometric functions, in Nonlinear Numerical Methods and Rational Approximation, Math. Appl., 43, 135- 142, Reidel, Dordrecht (1988).

10. G.V. Chudnovsky, Padé approximations to the generalized hypergeometric functions, J. Math. Pures Appl., 58 (1979), 445-476.

11. D.V. Chudnovsky, G.V. Chudnovsky, Padé and rational approximations to systems of functions and their arithmetic applications, in Lecture Notes in Mathematics, vol. 1052. Springer, Berlin, 1984.

12. A. Erdélyi, et al., Higher Transcendental Function, 1, McGraw Hill Book Co., Inc., New York, 1953.

13. M. Exton, Q-Hypergeometric Functions and Applications, Ellis Horwood series in mathematics and its applications, Chichester, 1983.

14. G. Gasper, M. Rahman, Basic hypergeometric series, Encyclopedia Mathematics and its Applications, 96, Cambridge university press, 2004

15. M. Hata, M. Huttner. Padé approximation to the logarithmic derivative of the Gauss hypergeometric function, Analytic number theory, 157-172, Springer, Boston, MA, 2002. 
16. C. Hermite, Sur la généralisation des fractions continues algébriques. Annali Math. Pura Appl., 21 (1893), 289-308.

17. A. Hurwitz, Uber arithmetische Eigenschaften gewisser transcendenter Funktionen. Math. Ann. 22 (1883), 211-229.

18. V. Kac, P. Cheung, Quantum calculus, Universitext series, IX, Springer Verlag, 2002.

19. T. Matala-aho, Type II Hermite-padé approximations of generalized hypergeometric series, Constr. Approx., 33 (2011), 289-312.

20. M.-L. Mazure, P.-J. Laurent, Affine and Non-affine Blossoms, in Torino Workshop on Computational Geometry, R. Conte, V. Demichelis, F. Fontanelle, I. Galligani (eds), World Scientific Pub. Singapore, 201-230, 1993.

21. M.-L. Mazure, Blossoming and CAGD algorithms, in Shape preserving representations for ComputerAided Design, Nova Science Pub., 99-117, 1999.

22. M.-L. Mazure, Blossoming: a geometrical approach, Constr. Approx., 15 (1999), 33-68.

23. V. Merilä, A nonvanishing lemma for certain Padé approximations of the second kind, Intern. J. Number Theory, 7 (2011), 1977-1997.

24. Y.V. Nesterenko (1994), Hermite-Padé approximants of generalized hypergeometric functions, Russ. Acad. Sci. Sb. Math. 83 (1995), 189-219.

25. H. Oruç, G.M. Phillips, $q$-Bernstein polynomials and Bézier curves, J. Comp. Appl. Math., 151 (2003), 1-12.

26. H. Pottmann, The geometry of Tchebycheffian splines, Comput. Aided Geom. Design, 10 (1993), 181-210.

27. L. Ramshaw, Blossoms are polar forms, Comput. Aided Geom. Design, 6 (1989), 323-358.

28. C. L. Seigel, Über einige Anwendungen diophantischer Approximationen, Abh. Preuss. Akad. Wiss., KL, 1, 1929.

29. P. Simeonov, V. Zafiris, R. Goldman, $q$-Blossoming: A new approach to algorithms and identities for $q$-Bernstein bases and $q$-Bézier curves, J. Approx. Theory, 164 (2012), 77-104

30. T. Stihl, Arithmetische Eigenschaften spezieller Heinescher Reihen, Math. Ann., 268 (1984), 21 ? 2.

31. A. Thue, Bemerkungen Über gewisse Näherungsbrüche algebraischer Zahlen, Kra. Vidensk. Selsk. Skrifter. I. Mat. Nat. K., 3 (1908).

32. A. Thue, Über rationale Annäherungswerte der reellen Würzel der ganzen Funktion dritten Grades $x^{3}-a x-b$, Kra. Vidensk. Selsk. Skrifter. I. Mat. Nat. M., 6 (1908).

33. W. Van Assche, Padé and Hermite-Padé Approximation and Orthogonality, Surveys in Approximation Theory, 2 (2006), 61-91.

34. M. Waldschmidt, Introduction to Diophantine methods: irrationality and transcendence, https://webusers.imj-prg.fr/ michel.waldschmidt/coursHCMUNS2007.html 